\title{
Design Principles for 2-Dimensional Molecular Aggregates using Kasha's Model: Tunable Photophysics in Near and Shortwave Infrared
}

Arundhati Deshmukh, Danielle Koppel, Chern Chuang, Danielle Cadena, Jianshu Cao, Justin Caram Submitted date: 17/05/2019 Posted date: 20/05/2019

Licence: CC BY-NC-ND 4.0

Citation information: Deshmukh, Arundhati; Koppel, Danielle; Chuang, Chern; Cadena, Danielle; Cao, Jianshu; Caram, Justin (2019): Design Principles for 2-Dimensional Molecular Aggregates using Kasha's Model: Tunable Photophysics in Near and Shortwave Infrared. ChemRxiv. Preprint.

Technologies which utilize near-infrared (700 - $1000 \mathrm{~nm})$ and short-wave infrared (1000 - $2000 \mathrm{~nm})$ electromagnetic radiation have applications in deep-tissue imaging, telecommunications and satellite telemetry due to low scattering and decreased background signal in this spectral region. It is therefore necessary to develop materials that absorb light efficiently beyond $1000 \mathrm{~nm}$. Transition dipole moment coupling (e.g. J-aggregation) allows for redshifted excitonic states and provides a pathway to highly absorptive electronic states in the infrared. We present aggregates of two cyanine dyes whose absorption peaks redshift dramatically upon aggregation in water from $\sim 800 \mathrm{~nm}$ to $1000 \mathrm{~nm}$ and $1050 \mathrm{~nm}$ respectively with sheet-like morphologies and high molar absorptivities $\left(e \sim 10^{5} \mathrm{M}^{-1} \mathrm{~cm}^{-1}\right)$. We use Frenkel exciton theory to extend Kasha's model for $\mathrm{J}$ and $\mathrm{H}$ aggregation and describe the excitonic states of 2-dimensional aggregates whose slip is controlled by steric hindrance in the assembled structure. A consequence of the increased dimensionality is the phenomenon of an intermediate "I-aggregate", one which redshifts yet displays spectral signatures of band-edge dark states akin to an $\mathrm{H}$-aggregate. We distinguish between $\mathrm{H}-, \mathrm{I}-$ and $\mathrm{J}$-aggregates by showing the relative position of the bright (absorptive) state within the density of states using temperature dependent spectroscopy. I-aggregates hold potential for applications as charge injection moieties for semiconductors and donors for energy transfer in NIR and SWIR. Our results can be used to better design chromophores with predictable and tunable aggregation with new photophysical properties. Technologies which utilize near-infrared $(700-1000 \mathrm{~nm})$ and short-wave infrared (1000 - 2000 $\mathrm{nm}$ ) electromagnetic radiation have applications in deep-tissue imaging, telecommunications and satellite telemetry due to low scattering and decreased background signal in this spectral region. It is therefore necessary to develop materials that absorb light efficiently beyond $1000 \mathrm{~nm}$. Transition dipole moment coupling (e.g. J-aggregation) allows for redshifted excitonic states and provides a pathway to highly absorptive electronic states in the infrared. We present aggregates of two cyanine dyes whose absorption peaks redshift dramatically upon aggregation in water from $\sim 800 \mathrm{~nm}$ to $1000 \mathrm{~nm}$ and $1050 \mathrm{~nm}$ respectively with sheet-like morphologies and high molar absorptivities $\left(e \sim 10^{5} \mathrm{M}^{-1} \mathrm{~cm}^{-1}\right)$. We use Frenkel exciton theory to extend Kasha's model for $\mathrm{J}$ and $\mathrm{H}$ aggregation and describe the excitonic states of 2-dimensional aggregates whose slip is controlled by steric hindrance in the assembled structure. A consequence of the 
increased dimensionality is the phenomenon of an intermediate "I-aggregate", one which redshifts yet displays spectral signatures of band-edge dark states akin to an $\mathrm{H}$-aggregate. We distinguish between $\mathrm{H}$-, Iand $\mathrm{J}$-aggregates by showing the relative position of the bright (absorptive) state within the density of states using temperature dependent spectroscopy. I-aggregates hold potential for applications as charge injection moieties for semiconductors and donors for energy transfer in NIR and SWIR. Our results can be used to better design chromophores with predictable and tunable aggregation with new photophysical properties.

File list (2)

2Daggs_main text_ChemRxiv.pdf (1.63 MiB) view on ChemRxiv - download file 
3 Aggregates using Kasha's Model: Tunable Photophysics

$7 \quad{ }^{\dagger}$ Department of Chemistry and Biochemistry, University of California, Los Angeles, 607 Charles E. Young Institute of Technology, Cambridge, Massachusetts 02139, United States. 
1 Abstract: Technologies which utilize near-infrared

$2 \quad(700-1000 \mathrm{~nm})$ and short-wave infrared (1000 -

$32000 \mathrm{~nm}$ ) electromagnetic radiation have

4 applications in deep-tissue imaging,

5 telecommunications and satellite telemetry due to

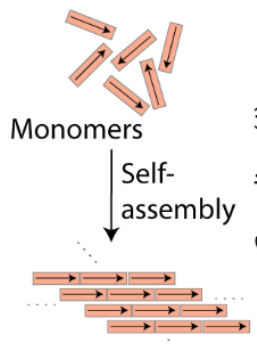

Large ordered sheets

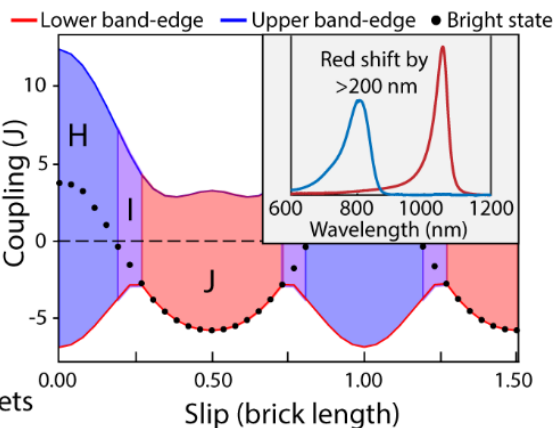

6 low scattering and decreased background signal in this spectral region. It is therefore necessary to develop materials that absorb light efficiently beyond $1000 \mathrm{~nm}$. Transition dipole moment coupling (e.g. Jaggregation) allows for redshifted excitonic states and provides a pathway to highly absorptive electronic states in the infrared. We present aggregates of two cyanine dyes whose absorption peaks redshift dramatically upon aggregation in water from $\sim 800 \mathrm{~nm}$ to $1000 \mathrm{~nm}$ and $1050 \mathrm{~nm}$ respectively with sheetlike morphologies and high molar absorptivities $\left(\varepsilon \sim 10^{5} \mathrm{M}^{-1} \mathrm{~cm}^{-1}\right)$. We use Frenkel exciton theory to extend Kasha's model for $\mathrm{J}$ and $\mathrm{H}$ aggregation and describe the excitonic states of 2-dimensional aggregates whose slip is controlled by steric hindrance in the assembled structure. A consequence of the increased dimensionality is the phenomenon of an intermediate "I-aggregate", one which redshifts yet displays spectral signatures of band-edge dark states akin to an $\mathrm{H}$-aggregate. We distinguish between $\mathrm{H}-, \mathrm{I}-$ and Jaggregates by showing the relative position of the bright (absorptive) state within the density of states using temperature dependent spectroscopy. I-aggregates hold potential for applications as charge injection moieties for semiconductors and donors for energy transfer in NIR and SWIR. Our results can be used to better design chromophores with predictable and tunable aggregation with new photophysical properties. 


\section{Introduction}

Near-infrared (NIR, $700-1000 \mathrm{~nm}$ ) and shortwave infrared (SWIR, 1000 - $2000 \mathrm{~nm}$ ) photoactive materials are highly sought out due to their superior performance in many applications, ranging from

4 deep-tissue imaging, ${ }^{1}$ to telecommunications, ${ }^{2}$ and LIDAR. ${ }^{3}$ Light in the NIR and SWIR transmits over

5 longer distances in the atmosphere and shows less loss for fiber-optic communication due to decreased

6 scatter and absorption in this spectral regime. ${ }^{2}$ In biomedical contexts, significantly decreased auto-

7 fluorescence from biomolecules in the SWIR promotes better signal-to-noise ratio in fluorescence 8 imaging. ${ }^{4-6}$

9 However, there are few molecular species (such as carbon nanotubes, lanthanide complexes and 10 flavylium polymethine dyes), which absorb efficiently beyond $1000 \mathrm{~nm} .{ }^{5,7}$ Approaches for designing strong 11 absorbers and emitters in NIR and SWIR are primarily based on covalent modification of conjugated dyes 12 and semiconductor nanocrystal materials. ${ }^{7-10}$ These materials cannot necessarily offer high molar 13 absorptivities and narrow linewidths, prerequisites for several technological applications such as 14 nonlinear optics and photonics. ${ }^{11,12}$ However, another approach to achieving strong SWIR absorption is 15 through coupling of multiple molecular transition dipole moments (TDMs, $\mu$ ) into extended excitonic 16 states. The oscillator strength and radiative rate of a molecule are proportional to the square of the TDM $17 \quad\left(\mu^{2}=\left|\left\langle g\left|\Sigma_{i} q_{i} r_{i}\right| e\right\rangle\right|^{2}\right.$, where $g$ and $e$ represent the ground and excited state wavefunctions, $q_{i}$ and $r_{i}$ are 18 the charges and their positions). ${ }^{13}$ In molecular aggregates, certain excitonic transitions are enhanced by 19 a nonlinear increase in the oscillator strength due to long-range coherent coupling among TDMs. ${ }^{14}$ 20 Molecular aggregates therefore can show increased absorption over a narrower wavelength range, 21 enhanced emission due to faster radiative rates, and long-range coherent energy transport. ${ }^{14-17}$ 
To begin, we define molecular aggregates as non-covalently bound molecular assemblies formed via

2 entropic and Van-der-Waals driving forces. ${ }^{14}$ Molecules within an aggregate undergo coherent TDM coupling, and electronic excitations extend over many molecules to form delocalized Frenkel excitons. ${ }^{18}$

The net excitonic TDM depends on the phase relationship of individual TDMs, their coupling- both defined by the geometric arrangement of dipoles- and the energetic disorder among the chromophores. ${ }^{14}$ Kasha developed a simple formalism describing how aggregation leads to absorption shifts for molecular dimers and linear chain aggregates as shown in Figure $1 a .{ }^{19}$ When dipoles are aligned in a head-to-tail fashion, the optically active bright state (with non-zero TDM) is also the lowest energy exciton. As a result, these arrangements give rise to a red shift in the absorption spectrum. On the other hand, cofacial dye organization renders the highest energy exciton as the only optically active state resulting in a blue shift

11 in the absorption spectrum. The blue-shifted aggregates are called $\mathrm{H}$-aggregates whereas the red-shifted 12 aggregates are called J-aggregates. ${ }^{14,20}$ One can shift between $\mathrm{H}$ - and J-aggregation by changing the slip 13 (or angle) between each dye monomer, and many groups have employed this approach to generate $\mathrm{H}$ - or J-aggregated structures. ${ }^{15,21}$ While the formalism for dimers and linear chains is well-known, ${ }^{14,19,22}$

a
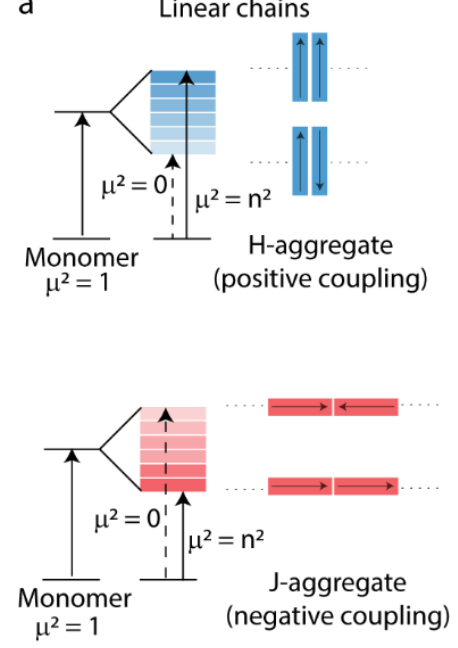

b 2-Dimensional sheets
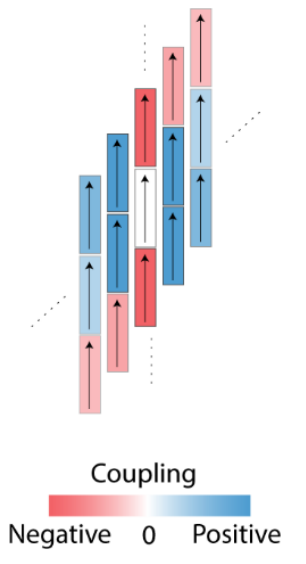
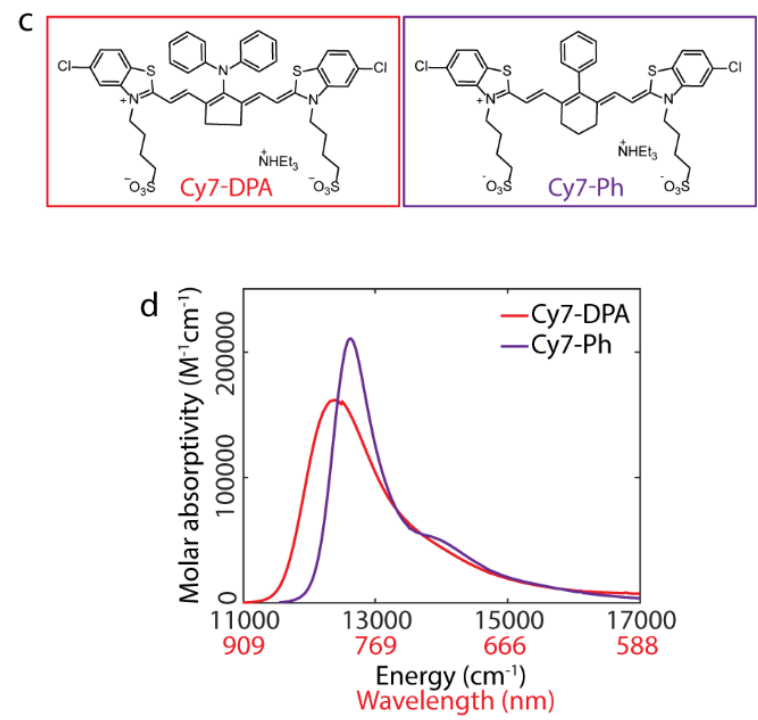

Figure 1. (a) Schematic depicting $\mathrm{H}$ - and J-aggregation for dimers and linear chain aggregates $(n=$ number of monomers in the chain) based on Kasha's model (Ref: 19), (b) A schematic depiction of coupling of a central brick (white) within a 2-dimensional aggregate with its neighbors, (c) Molecular structures of the cyanine dyes investigated, (d) Absorption spectra of the dyes in their monomeric form in methanol solutions. 
complications arise in the case of 2-dimensional (2D) or quasi-2D sheet and tubular aggregates. ${ }^{23}$ Along one axis, the predominant coupling will be negative (J-like) while coupling along the other axis could be positive (H-like). This is depicted as a cartoon in Figure $1 \mathrm{~b}$, where the coupling of the central brick (white) with its neighbors depends on the distance and the slip from the central. The net coupling would be determined by bricks along all directions. Even though several experimental ${ }^{24,25}$ and theoretical ${ }^{26,27}$ examples of 2D aggregates are known, general principles to tune absorption through control over molecular stacking during self-assembly are limited. In this manuscript, we show how controlling 2D aggregation slip-stacking can be used as an approach for further red shifting the absorption, and that additional control is needed to create emissive aggregates.

Many $\mathrm{H}$ - and J-aggregates have been reported based on small molecule chromophores like cyanines, ${ }^{15}$ perylene bisimides, ${ }^{28,29}$ porphyrins ${ }^{30,31}$ as well as for more extended structures like conjugated polymers, ${ }^{32}$ and pigment-proteins. ${ }^{14}$ Kasha's model and its variations ${ }^{32,33}$ have been critical in describing the rich photophysics in such systems. We focus on cyanine dyes - a class of dyes which consist of a polymethine bridge connecting to two aromatic heterocycles. Cyanine dyes are particularly interesting because of their tendency to self-assemble into different topologies including dimers, single and double-walled nanotubes, bundles and sheets. ${ }^{34-36}$ As a class, cyanine dyes exhibit large TDMs (as high as $10 \mathrm{D}$ ) and a high degree of structural and spectral tunability. ${ }^{15,37}$ Recently, long-range exciton migration was shown in doublewalled nanotubes of cyanine dyes in the visible region. ${ }^{38,39}$ Owing to these properties, cyanine dyes are extensively employed for biological imaging, Förster resonance energy transfer (FRET), nonlinear optics and photoredox reactions. ${ }^{3,4,40-42}$

In this paper, we exploit molecular aggregate structure and dimensionality to achieve new materials with high absorption cross sections above $1000 \mathrm{~nm}$. Through control of solvation conditions, we observe the aggregation of two NIR thiacarbocyanines dyes (Figure 1c) into multiple morphologies (like sheets, dimers) with unique spectral shifts. Here we focus on their most redshifted forms, which were found to 
1 be sheet-like structures with almost micron scale domains. These structures display strong $\left(\varepsilon \sim 10^{5} \mathrm{M}^{-}\right.$

$2{ }^{1} \mathrm{~cm}^{-1}$ ) SWIR absorption, though little to no direct emission. To connect the observed photophysics to

3 structure, we construct an analytical model based on long-range TDM coupling and calculate the density

4 of states (DOS) and other optical properties. Unique to the sheet morphology, we describe an 'I-aggregate'

5 one which exhibits intermediate photophysics between $\mathrm{J}$ - and $\mathrm{H}$-aggregates, a redshifting yet non-

6 emissive structure. Temperature dependent absorption spectroscopy experimentally confirms the model,

7 distinguishing the I- and J-aggregates. Finally, we relate our results to the dye structures and induced slip,

8 in analogy to Kasha's rules to elucidate design principles for controlling the photophysics of 2D aggregates.

$9 \quad$ Results and Discussions

11 ethylenethiatricarbocyanine, ammonium salt (Cy7-DPA) and 3,3'-bis(4-sulfobutyl)-5,5'-dichloro-11-

12 phenyl-10,12-ethylenethiatricarbocyanine, ammonium salt (Cy7-Ph) from FEW chemicals. Previous work
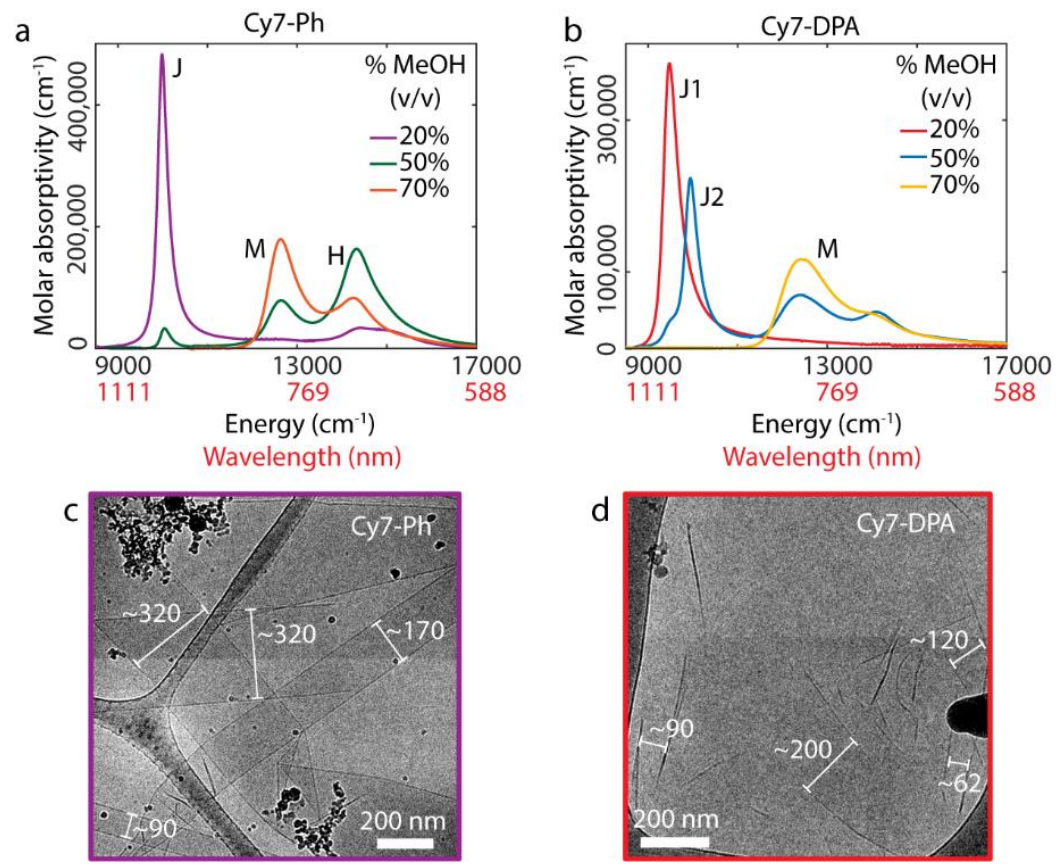

Figure 2. Top: Selected absorption spectra of (a) Cy7-Ph and (b) Cy7-DPA aggregates prepared by mixing methanol solutions of the dyes with deionized water with 20,50 and $70 \%$ methanol $(\mathrm{v} / \mathrm{v})$. Final dye concentration was kept constant $(0.2 \mathrm{mM})$ for all samples. $20 \%$ samples show a sharp redshifted peak in SWIR. Bottom: Cryo-electron micrographs of the most redshifted aggregates of (c) Cy7-Ph and (d) Cy7-DPA showing 2D sheet-like morphology. Numbers indicate sheet widths in nanometers. 
1 with similar cyanine dyes showed sheet-like aggregation in aqueous solutions, though detailed

2 photophysical and structural insights were not provided. ${ }^{35,43,44}$ Cy7-DPA and Cy7-Ph dyes, shown in Figure

3 1c, are structurally similar to each other apart from substitutions on their cyanine bridge which do not

4 significantly alter their monomer absorption (Figure 1d). This makes them optimal candidates for

5 comparing the effect of dye structure on aggregate formation and photophysics. Monomer absorption

6 peaks of Cy7-DPA and Cy7-Ph lie in NIR at $807 \mathrm{~nm}\left(12,391 \mathrm{~cm}^{-1}\right)$ and $792 \mathrm{~nm}\left(12,626 \mathrm{~cm}^{-1}\right)$ respectively.

7 Adapting procedures from previous literature, ${ }^{20,34}$ we explored the phase space of aggregation. Unlike

8 previous aggregation routes, which use direct dissolution of dye into water or brine, $\mathrm{pH}$ modification and

9 templating, $8,35,45,46$ we pre-dissolve the dyes in methanol prior to mixing with water following the so-called

10 "alcoholic route" to self-assembly. ${ }^{47}$ This procedure allows for independent control over methanol-water

11 ratios and dye concentration, and aggregates form relatively rapidly ( $24 \mathrm{hrs}$ ). In Figures $2 a-b$, we show

12 selected absorption spectra of aggregates where we fixed the dye concentration at $0.2 \mathrm{mM}$ while varying

13 the methanol-water ratios from 0-100\% MeOH v/v (complete range shown in Supporting Information, $\mathrm{SI}$

14 Figure S1). We observe sharp red-shifted J-aggregate peaks in SWIR at lower methanol-water ratios

$15(\% \mathrm{MeOH})$ for both the dyes. The J-aggregate peak of Cy7-DPA lies in SWIR at $\sim 1050 \mathrm{~nm}\left(9524 \mathrm{~cm}^{-1}\right)$ while that of Cy7-Ph lies at $1000 \mathrm{~nm}\left(10,000 \mathrm{~cm}^{-1}\right)$. As we increase the $\% \mathrm{MeOH}$, the J-aggregate peak decreases

17 and the monomer peak increases for both the dyes (SI Figure S1). At 50\% $\mathrm{MeOH}$, we observe an $\mathrm{H}$ 18 aggregate peak at $698 \mathrm{~nm}\left(14,327 \mathrm{~cm}^{-1}\right)$ for Cy7-Ph whereas Cy7-DPA shows a second J-aggregate peak 19 (J2) at $\sim 1000 \mathrm{~nm}\left(10,000 \mathrm{~cm}^{-1}\right)$ under the same conditions. We hypothesize that the diphenylamine (DPA) group in Cy7-DPA frustrates cofacial packing and as a result, we never observe an $\mathrm{H}$-aggregate peak in 21 Cy7-DPA.

We perform cryo-electron microscopy (cryoEM) on the most red-shifted samples. CryoEM of the $10 \%$ $\mathrm{MeOH}$ samples reveals sheet-like morphology with large planar domains extending over hundreds of 
1 like morphology and absorption lineshapes were observed for Cy5 and Cy7 thiacarbocyanine dye

2 aggregates. ${ }^{35,36}$ For a more global verification of domain sizes, we performed dynamic light scattering

3 (DLS). Estimates of domain sizes obtained from DLS agree well with cryoEM data with the smallest average

4 across multiple trials being $\sim 700 \mathrm{~nm}$ for Cy7-DPA and $\sim 900 \mathrm{~nm}$ for Cy7-Ph (SI Figure S2). Because of the

5 characteristic peak shape,,$^{35}$ we hypothesize that the $J 2$ peak on Cy7-DPA also arises from a sheet

6 morphology with slightly different dye packing. The shoulder on J2 is from some conversion to J1. From a

7 concentration dependence study (SI Section 2.2 and Figure S3), we assign the $\mathrm{H}$ peak of Cy7-Ph to a

8 dimer. $^{48}$

9 The presence of large domains and narrow linewidths suggests that the aggregates have long-range

delocalization of their transition dipole moments. We model the aggregate photophysics following the

11 Frenkel exciton model with extended dipole treatment developed by Kuhn and co-workers. ${ }^{49,50}$ First,

12 following convention, ${ }^{51}$ we consider a monomer as a brick with an internal dipole representative of the

13 TDM generated by a single excitation (Figure 3a). We then tile the bricks with a single variable parameter,

14 the slip $(s)$, which represents the displacement along the long axis and construct the sheet (Figure 3a).

15 Basing on the standard Frenkel exciton Hamiltonian, ${ }^{49}$

$$
H_{S}=\sum_{\boldsymbol{n} \neq \boldsymbol{m}} J(\boldsymbol{n}, \boldsymbol{m})|\boldsymbol{n}\rangle\left\langle\boldsymbol{m}\left|+\sum_{\boldsymbol{n}} \epsilon_{\boldsymbol{n}}\right| \boldsymbol{n}\right\rangle\langle\boldsymbol{n}|
$$

16 where $|\boldsymbol{n}\rangle$ represents the state where the $\boldsymbol{n}^{\text {th }}$ molecule is in the excited state while all others in the ground

17 state, $J(\boldsymbol{n}, \boldsymbol{m})$ the excitonic coupling between the two molecules, and $\epsilon_{\boldsymbol{n}}$ is the individual site energy.

18 Boundary effects are resolved by imposing periodic boundary conditions, $J(\boldsymbol{n}, \boldsymbol{m})=J(\boldsymbol{n}-\boldsymbol{m})$. We then

19 diagonalize the Hamiltonian, $H_{s}=\sum_{k} \epsilon_{k}|k\rangle\langle k|$, where $|k\rangle$ is the $k^{\text {th }}$ eigenstate with energy $\epsilon_{k}$. For planar

20 aggregates with one transition per unit cell, we obtain only one single state with finite oscillator strength

$21 \mu_{k}=\mu_{0}\left|\sum_{n}\langle k \mid n\rangle\right|$, representing when all dipoles are pointing the same direction. This is the state that

22 can absorb or emit light and is referred to as the bright state. All other excitonic states contain at least 
1 one node, and thus are optically dark. We further calculate the density of states (DOS), $D(E)=$

$2 \sum_{k} \delta\left(E-\epsilon_{k}\right)$, as well as the energy of the bright state as a function of slip (see SI section 3 and SI Figures

3 S4-S7 for details) for the 2D aggregates.

4 The results from this model are summarized in Figure $3 \mathrm{~b}$, where we set $\epsilon_{\boldsymbol{n}}=0$ for clarity. We observe,

5 for $0 \mathrm{~nm}$ slip, an $\mathrm{H}$-aggregate with the bright state near the upper band-edge. For higher slip values such

6 as $0.7 \mathrm{~nm}$, we observe a typical J-aggregate with a lower band-edge bright state. Interestingly, for

7 intermediate slip values like $0.4 \mathrm{~nm}$, the bright state is still red-shifted from the monomer, but notably,

8 not at the band-edge. We associate this case to an 'I-aggregate', or intermediate-aggregate as it displays

9 a red-shifted bright state like J-aggregates but has excitonic states below the bright state, similar to $\mathrm{H}$ -

10 aggregates. In other words, I-aggregates have a bright state which lies in the middle of the DOS but red-

11 shifted from the monomer. I- and J-aggregates cannot be distinguished simply from their absorption

12 spectra. We use their temperature dependent linewidth to determine where the bright state sits in the 13 density of states. 
Modeling 2-D aggregates

a

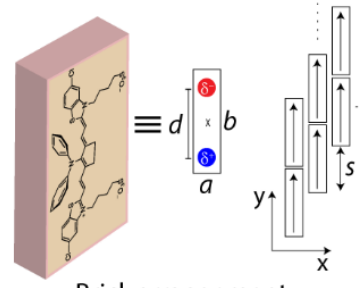

Brick arrangement

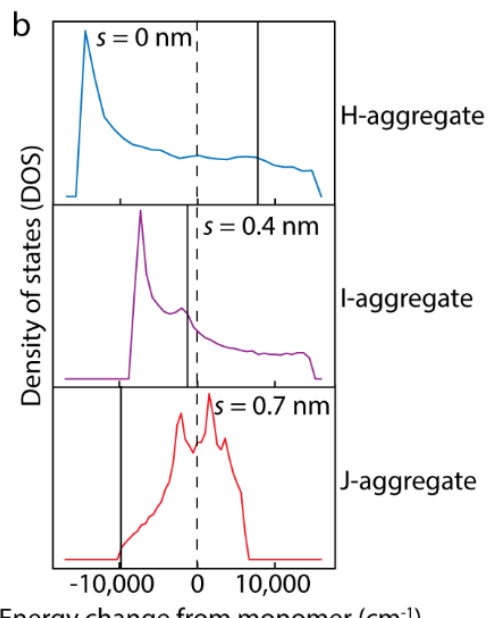

c

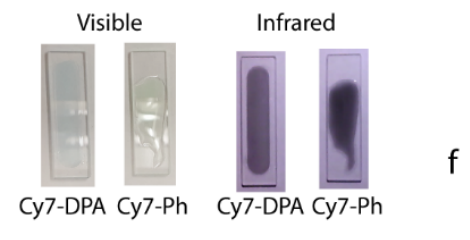

d

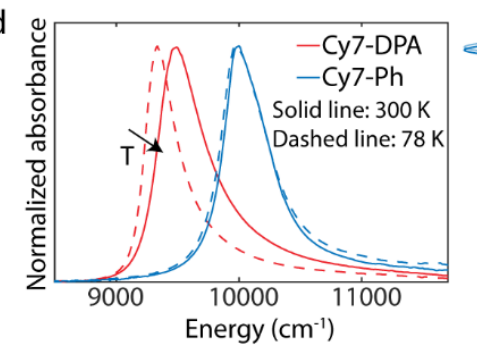

$e_{500}$

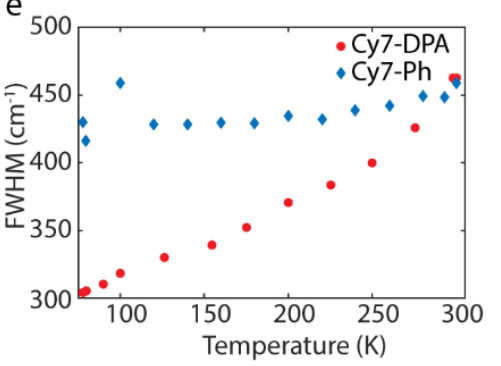

f

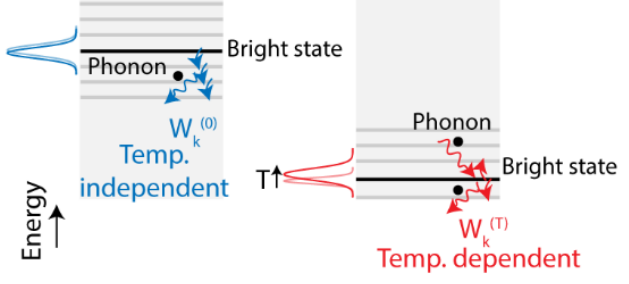

$\overline{\text { Ground state }} \quad \overline{\text { Ground state }}$

Cy7-DPA

Excitonic states in 2-D aggregates

Energy change from monomer $\left(\mathrm{cm}^{-1}\right)$

Figure 3. (a) Schematic depiction of the brick arrangement model; (b) Results from the analytical model showing monomer energy (dashed line) and relative position of the bright-state (solid line) within the density of states for 0 , 0.4 and $0.7 \mathrm{~nm}$ slips; (c) Pictures of sugar matrix stabilized aggregates as seen through a typical silicon camera with an IR filter (left) and with the IR filter removed and an $850 \mathrm{~nm}$ longpass filter added to remove stray light (right); (d) Absorption spectra of sugar matrix stabilized aggregates-Cy7-DPA (red) and Cy7-Ph (blue) at $300 \mathrm{~K}$ (solid lines) and $78 \mathrm{~K}$ (dashed lines); (e) Full-width at half-maximum (FWHM) of the absorption peak as a function of temperature for Cy7DPA (red circles) and Cy7-Ph (blue diamonds); (f) Schematic describing the processes that contribute to the lineshape of Cy7-Ph and Cy7-DPA.

We perform temperature dependent absorption spectroscopy on matrix stabilized Cy7-DPA and Cy7-

$\mathrm{Ph}$ aggregates $(20 \% \mathrm{MeOH})$. We prepare the stabilized aggregates using a previously reported sugar matrix stabilization procedure (pictures shown in Figure 3c) ${ }^{39,52}$ Comparison of solution and sugar matrix stabilized aggregates (shown in SI Figure S8a) strongly suggests that the aggregate morphology remains intact in sugar matrix. Upon cooling down from room temperature to $78 \mathrm{~K}$, we find that the full-width at half-maximum (FWHM) of Cy7-DPA peak narrows from $463 \mathrm{~cm}^{-1}$ to $303 \mathrm{~cm}^{-1}(34 \%)$ and red shifts by 150 $\mathrm{cm}^{-1}$ as shown in Figures 3d-e. On the other hand, absorption of Cy7-Ph barely changes upon cooling. The peak position of Cy7-DPA is similarly sensitive to temperature as it red-shifts upon cooling while that of Cy7-Ph barely changes (SI Figure S8b). 
The effect of temperature on absorption lineshape is described by the origin of fluctuations that

2 dephase the ground-excited state coherence. The absorption spectrum is the Fourier transform of the 3 transition dipole autocorrelation function, ${ }^{22}$

$$
A(t)=\langle a(t)\rangle \approx\left\langle e^{-i \epsilon_{\mathrm{b}} t-W_{\mathrm{b}} t}\right\rangle
$$

4 where $\epsilon_{\mathrm{b}}$ is the energy gap between ground and excited state and $W_{\mathrm{b}}$ represents how the system's

5 interaction with the bath leads to energetic fluctuations that dephase the phase relationship between

6 ground and excited state wavefunctions. The brackets represent averaging over inhomogeneous disorder,

7 or fluctuations in the environmental degrees of freedom slower than the lifetime of the exciton. Using the

8 Redfield framework for exciton dynamics, and imposing the secular approximation and Markovian bath,

9 we express $W_{\mathrm{b}}$ for each exciton as follows: ${ }^{53-55}$

$$
W_{k}=\sum_{l \neq k} \frac{O_{k l}}{e^{\left(\epsilon_{l}-\epsilon_{k}\right) / k_{\mathrm{B}} T}-1} D_{b}\left(\epsilon_{l}-\epsilon_{k}\right)
$$

10 where $O_{k l}=\sum_{n}|\langle k \mid n\rangle\langle n \mid l\rangle|^{2}$ is the wavefunction overlap between the excitonic states, $k_{\mathrm{B}}$ is the

11 Boltzmann constant, and $D_{b}(E)=-D_{b}(-E)$ is the antisymmetrized bath spectral density. For

12 homogeneous, translational invariant systems the secular approximation is by construct exact. ${ }^{51}$ On the 13 other hand, Markovian approximation is justified for fast dissipating environment. While this is not

14 necessarily true for the systems under consideration, as exemplified by the existence of vibronic 15 progression observed in both monomer spectra (Figure 1d), the line width and its temperature 16 dependence are well captured by the Markovian contribution. ${ }^{49}$ While the inhomogeneous contribution

17 to the linewidth is mostly temperature independent, one can further breakdown the homogeneous 18 linewidth (or dephasing rate) $W_{k}$ into (i) stimulated absorption/emission of phonons and (ii) spontaneous 19 emission of phonons and relaxation to band-edge. 


$$
\begin{array}{r}
W_{k}=\sum_{l \neq k} \frac{O_{k l}}{e^{\left|\epsilon_{l}-\epsilon_{k}\right| / k_{\mathrm{B}} T}-1} D_{b}\left(\left|\epsilon_{l}-\epsilon_{k}\right|\right) \\
+\sum_{\epsilon_{l}<\epsilon_{k}} O_{k l} \cdot D_{b}\left(\epsilon_{k}-\epsilon_{l}\right) \\
=W_{k}^{(\mathrm{T})}+W_{k}^{(0)}
\end{array}
$$

Here, $W_{k}^{(\mathrm{T})}$ represents the stimulated absorption/emission portion, and thus depends on the thermal

2 occupation of phonons (making it temperature dependent) whereas the $W_{k}^{(0)}$ relies on the available DOS

3 below the bright state and is temperature independent. ${ }^{31}$ In Cy7-Ph aggregates, we observe minimal

$4 \quad$ change with temperature implying that the second term (relaxation to band-edge through spontaneous

5 emission of phonons) is more dominant in the lineshape function. This implies sufficient DOS below the

6 bright state, matching the description of an I-aggregate (Figure 3b). On the other hand, we observe a

7 significant narrowing upon cooling in Cy7-DPA which suggests that the first term dominates the lineshape

8 function. Therefore, Cy7-DPA must have a bright state that is thermally accessible from the band-edge.

9 We explain this in Figure $3 f$ where the left side shows spontaneous emission of a phonon and the

10 subsequent relaxation to the lower energy state. This process is governed by the term $\mathrm{W}_{\mathrm{k}}^{(0)}$ of equation

11 (4) which is temperature independent. Right side of Figure $3 \mathrm{f}$ shows stimulated emission of phonons,

12 governed by $\mathrm{W}_{\mathrm{k}}{ }^{(\mathrm{T})}$ which depends on the thermal occupation of phonons. As a result, the spectrum shown

13 on the left (blue) doesn't change with temperature while the one on right (red) broadens with increasing

14 temperature. The lineshape of Cy7-DPA is dominated by $W_{k}^{(T)}$ and hence, narrows down significantly with

15 decreasing temperature. The lineshape of Cy7-Ph, on the other hand, is dominated by $W_{k}^{(0)}$ and therefore,

16 does not change with temperature matching our experimental observations (Figure 3d). Simply put, when

17 the bright state is near the band-edge, it's lineshape will be temperature dependent. As it moves away

18 from the band-edge, the lineshape is dominated by temperature independent relaxation within the 
density of states. Despite both Cy7-Ph and Cy7-DPA appearing to "J-aggregate" from their absorption spectra, the temperature dependence strongly suggests that the bright state is not at the band-edge.

Recalling Figure $3 b$, the differences in aggregation arise due to the slip parameter. To correlate the structural parameters (slip) and spectroscopic observables, we calculate the DOS and bright state for a generalized $1 \mathrm{D}$ and $2 \mathrm{D}$ aggregates as a function of slip (Figure 4a). We use a set of general parameters shown in Table 1, which represent the average of calculations used to determine the extended dipole parameters for Cy7-DPA and Cy7-Ph (SI section 3.4). We express the coupling in terms of the variable J, defined as the coupling of a nearest neighbor head-to-tail dimer $\left(J=-2000 \mathrm{~cm}^{-1}\right)$ calculated using extended dipole model (equation (3) in the SI), and the slip in terms of the length of the brick $(b)$. The maximum and minimum eigenvalues define the upper and lower band-edge of the DOS and the region between the two extremes represents the exciton bandwidth. As seen from Figure 4a, 1D aggregates follow Kasha's

a

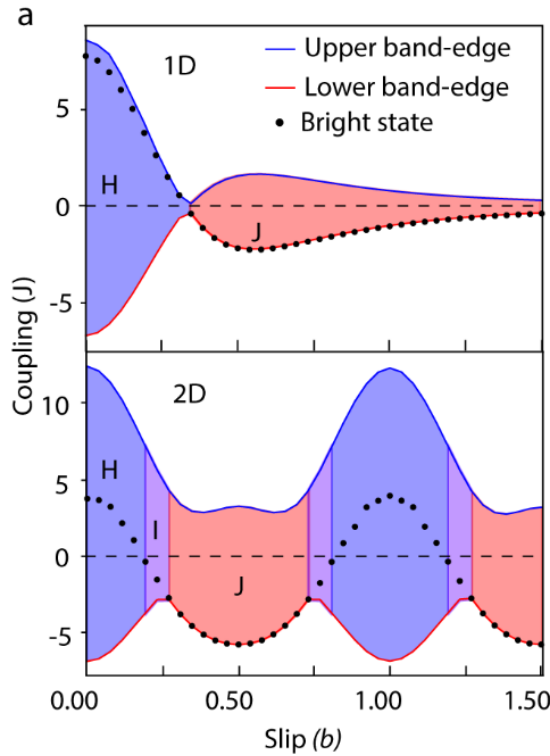

H
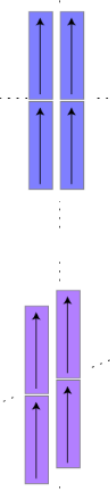

$J$

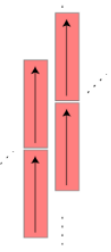

b

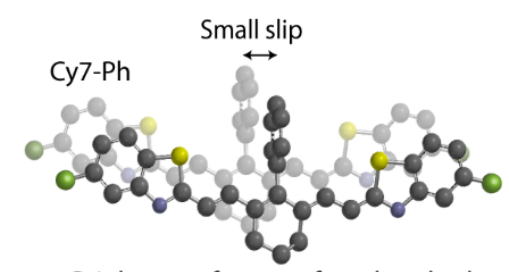

Bright state far away from band-edge

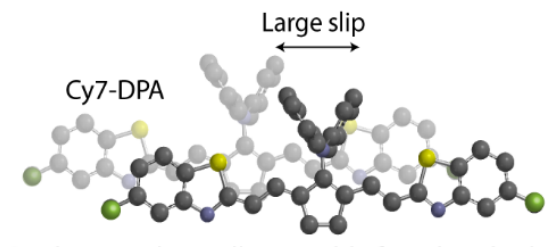

Bright state thermally accesible from band-edge

Design principles for 2D molecular aggregates

Figure 4. (a) Bright state (black dots), upper (blue) and lower (red) band-edge of the excitonic band as a function of slip for $1 D$ (top) and $2 D$ (bottom) aggregates with non-nearest neighbor coupling. $J$ is coupling for a head-to-tail dimer and $b$ is the length of the brick. (b) Schematic depiction of molecular design principles for 2D aggregates (alkyl chains have been omitted for simplicity). Packing with higher slip results in a bright state closer to the band-edge. 
aggregates we observe regions $\mathrm{H}-, \mathrm{I}$ - and J- aggregation (color-coded blue, purple and red respectively in

2 Figure 4a) based on the position of the bright state within the DOS. Furthermore, 2D aggregates display

3 increased possible spectral shifts due to stronger coupling. This unique band structure is a direct

4 consequence of the 2D topology (shown in SI Figure S9).

5 Table 1. List of parameters used in the model, $\boldsymbol{a}$ and $\boldsymbol{b}$ are the short and long axes of the brick respectively, $\boldsymbol{\mu}$ is the 6 transition dipole moment and $\boldsymbol{d}$ is the charge distance for the extended dipole (See SI for section 3 for details).

\begin{tabular}{|l|l|l|l|l|}
\hline & $a(\AA)$ & $b(\AA)$ & $\mu(D)$ & $d(\AA)$ \\
\hline Cy7-DPA & 7.3 & 19 & 4.2 & 9 \\
\hline Cy7-Ph & 8.8 & 22 & 4.0 & 3.5 \\
\hline Generalized & 8.1 & 21 & 4.1 & 6.3 \\
\hline
\end{tabular}

7

Similar behavior plays an important role in conjugated polymer photophysics ( $\mathrm{HJ}$ aggregates) wherein, changing the relative strength of intra-/interchain coupling gives a mid-band bright state. ${ }^{56,57}$ While polymer networks involve covalent coupling along the polymer backbone and dipole-dipole coupling between the polymer chains, their absorption spectra have strong analogies to the Van-der-Waals aggregates described here. Furthermore, epitaxially grown 2D crystals of a perylene based dye also resulted in a similar observation where limited aggregate size with non-unity aspect ratios gave dark bandedge J-aggregates. ${ }^{24}$ In this case, even with a dark band-edge, the next higher energy state was bright. Due to the extended nature of these $2 \mathrm{D} \mathrm{I}$-aggregates, the bright state position in the density of states is a nearly continuous function of the slip as seen from Figure $4 a$, and is thus tunable using chemical modification. Size control of self-assembled aggregates is challenging in solution. However, many SWIR applications demand solution aggregates for processability and biological compatibility. Our approach enables chemical control of the slip while also being more versatile.

Another recent theoretical investigation of 2D aggregates based on a simple dipole model demonstrates that temperature dependent peak shifts in absorption can be related back to structural 
1 parameters like slip but they observe I-aggregate like behavior at higher slips. ${ }^{58}$ The temperature

2 dependent behavior of the bright state is dominated by the short-range interactions which depend

3 strongly on the type of Hamiltonian used. Cy7-Ph shows little change in peak position while Cy7-DPA blue-

4 shifts with increasing temperature, consistent with having different slip parameters (SI Figure S8b). Using

5 our classification scheme, we estimate that the slip for the Cy7-Ph is $\sim 4-5 \AA$ while the slip for the Cy7-DPA

6 is between 7-10 $\AA$. In general, the steric hindrance due to the DPA group will prohibit slips that are less

7 than $7 \AA$ (which correlates to 0.36 times its brick length), consistent with our observation that Cy7-DPA

8 has a bright state closer to the band-edge than Cy7-Ph (Figure 4b). It is difficult to quantitatively compare

9 our model to the experiment owing to the complexities arising from modeling the structural nature of disorder and variance in the system dielectric. ${ }^{59-61}$

Using an approximate slip parameter of Cy7-Ph we introduce diagonal disorder to fit the absorption

12 FWHM and estimate the number of sites that participate in a given exciton. Focusing on Cy7-Ph which has

13 a narrower range of likely slip parameters, we calculate the linewidth for a range of disorder values and

14 compare to our experimental spectra. From this, we estimate that the diagonal disorder is $\sim 200 \mathrm{~cm}^{-1}$, and

15 the inverse participation value is $\sim 8$ (SI Figure S10). Despite relatively strong disorder, the high degree of

16 coupling in a 2D aggregate enables long-range delocalization lengths even at room temperature. This

17 strongly implies that a band picture is appropriate in describing the relevant photophysics (SI Figure S9).

\section{Conclusion}

We conclude by suggesting that in direct analogy to 1D aggregates described by Kasha's model, 2D

brick-like aggregates can be tuned through control of the slip parameter. In general, in 2D aggregates, a

21 slip value less than 0.18 times the brick length forms an $\mathrm{H}$-aggregate while a slip value greater than 0.26

22 times the brick length produces a J-aggregate. Interestingly, the remaining intermediate values result in 
1 I-aggregation which shows characteristics of both J-and $\mathrm{H}$ - structures. The observed spectroscopic

2 behavior of Cy7-Ph and Cy7-DPA is thus directly correlated to dye structures.

Our results demonstrate the significance of aggregate morphology and packing as a new avenue for tuning excitonic properties. In general, 2D aggregates with strong SWIR absorption can be made by simply tuning the solvation environment of NIR dyes. However, for 2D aggregates, the position of the bright state depends on the slip and is not restricted to the band-edge unlike linear or dimer aggregate systems allowing for emergent photophysics. Using temperature dependence, we confirm that Cy7-Ph forms an Iaggregate with dark states below the bright state. Cy7-DPA also forms an I-aggregate but has a bright

9 state closer to the band-edge because of higher slip induced by the bulkier DPA group. This may explain why both the aggregates were not emissive. Nevertheless, 2D aggregates follow a generalized

11 classification scheme which provides a roadmap to designing custom chromophore assemblies with

12 desired properties. For example, the central position on the cyanine bridge may be further modified with 13 bulkier groups to induce greater slips between chromophores to achieve band-edge J-aggregates.

We can exploit the 2D topology to access unique excitonic properties and employ them for NIR and SWIR antennas. Wang et al. demonstrated enhanced FRET efficiency between a quantum dot pair when mediated by a SWIR absorbing J-aggregate. ${ }^{40}$ This occurs despite the apparent low quantum yield of the

17 aggregate suggesting I-aggregate mediated transport. I-aggregates present the possibility of energy 18 transfer via dark states that can be accessed via near-field coupling similar to LH2 complex in 19 photosynthetic bacteria. ${ }^{62}$ Recently, a theoretical study showed dark states in $2 \mathrm{D}$ aggregates can be accessed via near-field coupling. ${ }^{63}$ Anantharaman et al. reported platelets of another Cy3 dye which show

21 very small narrowing with decreasing temperature, suggesting that the platelets might be I-aggregates. ${ }^{45}$

22 The interesting aspect of I-aggregates- a red-shifted bright state far away from the band-edge- provides opportunities for NIR/SWIR absorption and charge injection into another semiconductor in photovoltaics

24 enabling a complete utilization of the solar spectrum ${ }^{32,64,65}$ as well as for exploring fast energy transfer 
1 from I-aggregates to enhance the emission of NIR/SWIR materials. ${ }^{66}$ Recent studies have shown deep-

2 tissue imaging of mice vasculature using flavylium polymethine dyes with monomeric SWIR emission. ${ }^{7}$

3 Chemical modification of such dyes following the principles outlined here could enable J-aggregation,

4 pushing them deeper into the SWIR with high molar absorptivity needed for low dosage amounts.

5 Furthermore, these materials open the possibilities for exciton-polariton coupling at telecom relevant

6 wavelengths for antennas and other nanoscale optical devices. ${ }^{67}$

\section{Experimental Section}

8 Materials: Cy7-DPA and Cy7-Ph were obtained from FEW chemicals GmbH (catalog no. S0837 and S2433).

9 Spectroscopic grade methanol, sucrose and D-(+)-trehalose were obtained from ThermoFisher Scientific.

10 All materials were used as obtained, without any further purification. Deionized water (18 $\mathrm{M} \Omega$ ) was used

11 for making all samples.

12 Sample preparation: Cuvettes and sample vials were pre-soaked in deionized water for hydrophilization.

13 Dyes were pre-dissolved in methanol to make different concentrations of monomer solution. The

14 monomer solution was then added to deionized water in a specific volume/volume ratio to prepare the

15 aggregate solution. All solutions were stored in foil covered and parafilm sealed vials for 24 hrs before

16 taking any measurements. For scanning the whole \% $\mathrm{MeOH}$ range (10-100\%) while keeping the dye

17 concentration constant, the starting concentration of monomer solution was adjusted accordingly for

18 each sample. For concentration dependence of the $\mathrm{H}$-aggregate, $\% \mathrm{MeOH}$ was kept constant at $70 \%$ white

19 the final concentration was varied (0.05-0.5 mM). Samples for temperature dependence were prepared

20 by sugar-matrix stabilization method previously reported..$^{39}$ Dye aggregates were first prepared in water-

21 methanol solutions as mentioned above and stored for $24 \mathrm{hrs}$ to let the aggregates assemble. A saturated

22 sugar solution was made by dissolving a 50:50 sucrose:trehalose (wt./wt.) mixture in distilled water. To a

$23100 \mu \mathrm{L}$ aggregate solution, $100 \mu \mathrm{L}$ of the saturated sugar solution was added slowly and gently mixed. This 
1 solution was drop-cast onto a $0.2 \mathrm{~mm}$ quartz cuvette and kept under vaccum in dark for 24 hrs. CryoEM

2 samples were prepared on mesh 200 lacey formvar/carbon copper grids obtained from Ted Pella Inc. The

3 grids were plasma cleaned under a $\mathrm{H}_{2} / \mathrm{O}_{2}$ gas flow using Solarus Gatan Plasma cleaner for hydrophilization.

$4 \quad$ Vitrobot Mark IV was used for plunge freezing the samples. About 3.5 $\mu \mathrm{L}$ of the aggregate solution was

5 dropped onto the grid, excess solution was removed by blotting for $3.0 \mathrm{~s}$ with standard blotting paper

6 from Ted Pella and immediately dropped into liquid ethane maintained close to its freezing point using

7 liquid nitrogen. The frozen grids were stored in liquid nitrogen.

8 Measurements: All spectra were taken in a $0.2 \mathrm{~mm}$ path length quartz cuvette obtained from Starna Cells

9 Inc. All room temperature absorption spectra were taken on a JASCO V-770 UV/Vis/NIR spectrometer.

10 Temperature dependent absorption measurements were performed using a Shimadzu UV/Vis/NIR

11 spectrometer inside a liquid nitrogen cooled Janis ST-100 cryostat. Lakeshore 330 Autotuning

12 Temperature Controller was used to control the temperature. CryoEM images were recorded on a FEI

13 TF20 electron microscope equipped with a field emission gun at $200 \mathrm{kV}$. CryoEM grids were loaded on to

14 a Gatan 626 cryo-transfer sample holder, then inserted into the microscope and images were taken, all

15 under liquid nitrogen. Images were recorded on a CCD camera with $4 \mathrm{k}$ x $4 \mathrm{k}$ resolution. Image defocus was

16 used to enhance contrast. DLS experiments were performed on a Coulter Beckman Dynamic Light

17 Scattering Analyzer in $1 \mathrm{~cm}$ path length cuvettes.

18 ASSOCIATED CONTENT

19 Supporting Information. Aggregation in methanol-water mixtures, DLS measurements and analysis,

20 details of the model construction, sugar matrix stabilization, temperature dependent peak shifts,

21 estimates of exciton delocalization, and band structure for 1D and 2D aggregates. This material is available

22 free of charge via the Internet at http://pubs.acs.org.

\section{AUTHOR INFORMATION}


$2 *$ jearam@chem.ucla.edu

3 ORCID:

4 Arundhati P. Deshmukh: 0000-0002-7901-8814

5 Justin R. Caram: 0000-0001-5126-3829

\section{Author Contributions}

7 APD did all the experiments with help from DC and DK performed all the computations with the help of

8 CC. All authors contributed to writing the manuscript

$9 \quad$ Notes

10 Authors declare no conflict of interest.

\section{ACKNOWLEDGMENT}

12 We thank Prof. Ellen Sletten and Materials Characterization Laboratory at UCLA for instrumentation. We

13 acknowledge Doran I. G. Bennet for input towards 'I-aggregate' terminology. DC thanks Clare Booth Luce

14 Fellowship for funding. We thank the UCLA Academic Senate for Faculty Research Grant. The authors

15 acknowledge the use of instruments at the Electron Imaging Center for NanoMachines supported by NIH

16 (1S10RR23057 to ZHZ) and CNSI at UCLA.

17 REFERENCES

19 (1) Chen, W.; Cheng, C.; Cosco, E.; Ramakrishnan, S.; Lingg, J.; Bruns, O.; Zink, J. I.; Sletten, E.

20 Shortwave Infrared Imaging with J-Aggregates Stabilized in Hollow Mesoporous Silica

21 Nanoparticles. ChemRxiv 2018, DOI: 10.26434/chemrxiv.7503506.v1. 
1 (2) Hansen, M. P.; Malchow, D. S. Overview of SWIR Detectors, Cameras, and Applications. Proc. SPIE

2008, 6939, 69390I.

(3) Bouit, P. A.; Wetzel, G.; Berginc, G.; Loiseaux, B.; Toupet, L.; Feneyrou, P.; Bretonnière, Y.; Kamada, K.; Maury, O.; Andraud, C. Near IR Nonlinear Absorbing Chromophores with Optical Limiting Properties at Telecommunication Wavelengths. Chem. Mater. 2007, 19 (22), 5325-5335.

(4) Carr, J. A.; Franke, D.; Caram, J. R.; Perkinson, C. F.; Saif, M.; Askoxylakis, V.; Datta, M.; Fukumura, D.; Jain, R. K.; Bawendi, M. G.; Bruns, O. T. Shortwave Infrared Fluorescence Imaging with the Clinically Approved Near-Infrared Dye Indocyanine Green. Proc. Natl. Acad. Sci. U. S. A. 2018, 115 (17), 4465-4470.

(5) Thimsen, E.; Sadtler, B.; Berezin, M. Y. Shortwave-Infrared (SWIR) Emitters for Biological Imaging: A Review of Challenges and Opportunities. Nanophotonics 2017, 6 (5), 1043-1054.

(6) Hong, G.; Antaris, A. L.; Dai, H. Near-Infrared Fluorophores for Biomedical Imaging. Nature Biomedical Engineering. Nature Publishing Group January 10, 2017, p 0010.

(7) Cosco, E. D.; Caram, J. R.; Bruns, O. T.; Franke, D.; Day, R. A.; Farr, E. P.; Bawendi, M. G.; Sletten, E. M. Flavylium Polymethine Fluorophores for Near- and Shortwave Infrared Imaging. Angew. Chemie - Int. Ed. 2017, 56 (42), 13126-13129.

(8) Wang, C.; Weiss, E. A. Sub-Nanosecond Resonance Energy Transfer in the Near-Infrared within Self-Assembled Conjugates of PbS Quantum Dots and Cyanine Dye J-Aggregates. J. Am. Chem. Soc. 2016, $138(30)$, 9557-9564.

(9) Levitz, A.; Marmarchi, F.; Henary, M. Introduction of Various Substitutions to the Methine Bridge of Heptamethine Cyanine Dyes: Via Substituted Dianil Linkers. Photochem. Photobiol. Sci. 2018, $17(10), 1409-1416$. 
(10) Bricks, J. L.; Kachkovskii, A. D.; Slominskii, Y. L.; Gerasov, A. O.; Popov, S. V. Molecular Design of near Infrared Polymethine Dyes: A Review. Dye. Pigment. 2015, 121, 238-255.

(11) Boyd, G. T. Applications Requirements for Nonlinear-Optical Devices and the Status of Organic Materials. J. Opt. Soc. Am. B 1989, 6 (4), 685.

(12) Thomas, R.; Thomas, A.; Pullanchery, S.; Joseph, L.; Somasundaran, S. M.; Swathi, R. S.; Gray, S. K.; Thomas, K. G. Plexcitons: The Role of Oscillator Strengths and Spectral Widths in Determining Strong Coupling. ACS Nano 2017, 12 (1), 402-415.

(13) Hilborn, R. C. Einstein Coefficients, Cross Sections, f Values, Dipole Moments, and All That. Am. J. Phys. 1982, 50, 982.

(14) Brixner, T.; Hildner, R.; Köhler, J.; Lambert, C.; Würthner, F. Exciton Transport in Molecular Aggregates - From Natural Antennas to Synthetic Chromophore Systems. Adv. Energy Mater. 2017, $7(16), 1700236$.

(15) Bricks, J. L.; Slominskii, Y. L.; Panas, I. D.; Demchenko, A. P. Fluorescent J-Aggregates of Cyanine Dyes: Basic Research and Applications Review. Methods Appl. Fluoresc. 2017, 6 (1), 012001.

(16) Doria, S.; Sinclair, T. S.; Klein, N. D.; Bennett, D. I. G.; Chuang, C.; Freyria, F. S.; Steiner, C. P.; Foggi, P.; Nelson, K. A.; Cao, J.; Aspuru-Guzik, A.; Lloyd, S.; Caram, J. R.; Bawendi, M. G. Photochemical Control of Exciton Superradiance in Light-Harvesting Nanotubes. ACS Nano 2018, $12(5), 4556-4564$.

(17) Tong Zhu, Yan Wan, L. H. Direct Imaging of Frenkel Exciton Transport by Ultrafast Microscopy. Acc. Chem. Res. 2017, 50, 1725-1733.

(18) Scholes, G. D.; Rumbles, G. Excitons in Nanoscale Systems. Nat. Mater. 2006, 5 (11), 920-920.

(19) Kasha, M. Energy Transfer Mechanisms and the Molecular Exciton Model for Molecular 
Aggregates. Radiat. Res. 1963, 20 (1), 55-71.

(20) Jelley, E. E. Spectral Absorption and Fluorescence of Dyes in the Molecular State. Nature 1936, 138 (3502), 1009-1010.

(21) Kaiser, T. E.; Stepanenko, V.; Würthner, F. Fluorescent J-Aggregates of Core-Substituted Perylene Bisimides: Studies on Structure-Property Relationship, Nucleation-Elongation Mechanism, and Sergeants-and-Soldiers Principle. J. Am. Chem. Soc. 2009, 131 (19), 6719-6732.

(22) May, V; Kühn, O. Charge and Energy Transfer Dynamics in Molecular Systems, 3rd ed.; WileyVCH:Berlin, 2011.

(23) Nabetani, A.; Tomioka, A.; Tamaru, H.; Miyano, K. Optical Properties of Two-dimensional Dye Aggregate. J. Chem. Phys. 1995, 102 (13), 5109-5117.

(24) Eisfeld, A.; Marquardt, C.; Paulheim, A.; Sokolowski, M. Superradiance from Two Dimensional Brick-Wall Aggregates of Dye Molecules: The Role of Size and Shape for the Temperature Dependence. Phys. Rev. Lett. 2017, 119 (9), 097402.

(25) Prokhorov, V. V; Perelygina, O. M.; Pozin, S. I.; Mal'tsev, E. I.; Vannikov, A. V. Polymorphism of Two-Dimensional Cyanine Dye J-Aggregates and Its Genesis: Fluorescence Microscopy and Atomic Force Microscopy Study. J. Phys. Chem. B 2015, 119 (48), 15046-15053.

(26) Möbius, D.; Kuhn, H. Energy Transfer in Monolayers with Cyanine Dye Sheibe Aggregates. J. Appl. Phys. 1988, 64 (10), 5138-5141.

(27) Bakalis, L. D.; Rubtsov, I.; Knoester, J. Absorption Spectra of Mixed Two-Dimensional Cyanine Aggregates on Silver Halide Substrates. J. Chem. Phys. 2002, 117 (11), 5393-5403.

(28) Wolter, S.; Magnus Westphal, K.; Hempel, M.; Würthner, F.; Kühn, O.; Lochbrunner, S. Low Temperature Exciton Dynamics and Structural Changes in Perylene Bisimide Aggregates. J. Chem. 
Phys. 2017, 50 (18), 184005.

(29) Kaufmann, C.; Kim, W.; Nowak-Król, A.; Hong, Y.; Kim, D.; Würthner, F. Ultrafast Exciton Delocalization, Localization, and Excimer Formation Dynamics in a Highly Defined Perylene Bisimide Quadruple $\pi$-Stack. J. Am. Chem. Soc. 2018, jacs.7b11571.

(30) Wan, Y.; Stradomska, A.; Knoester, J.; Huang, L. Direct Imaging of Exciton Transport in Tubular Porphyrin Aggregates by Ultrafast Microscopy. J. Am. Chem. Soc. 2017, 139 (21), 7287-7293.

(31) Wan, Y.; Stradomska, A.; Fong, S.; Guo, Z.; Schaller, R. D.; Wiederrecht, G. P.; Knoester, J.; Huang, L. Exciton Level Structure and Dynamics in Tubular Porphyrin Aggregates. J. Phys. Chem. C 2014, $118(43), 24854-24865$.

(32) Hestand, N. J.; Spano, F. C. Molecular Aggregate Photophysics beyond the Kasha Model: Novel Design Principles for Organic Materials. Acc. Chem. Res. 2017, 50 (2), 341-350.

(33) Zheng, C.; Zhong, C.; Collison, C. J.; Spano, F. C. Non-Kasha Behavior in Quadrupolar Dye Aggregates: The Red-Shifted H-Aggregate. J. Phys. Chem. C 2019, 123 (5), 3203-3215.

(34) Eisele, D. M.; Arias, D. H.; Fu, X.; Bloemsma, E. A.; Steiner, C. P.; Jensen, R. A.; Rebentrost, P.; Eisele, H.; Tokmakoff, A.; Lloyd, S.; Nelson, K. A.; Nicastro, D.; Knoester, J.; Bawendi, M. G. Robust Excitons Inhabit Soft Supramolecular Nanotubes. Proc. Natl. Acad. Sci. 2014, 111 (33), E3367E3375.

(35) Berlepsch, H. V; Böttcher, C. Tubular J-Aggregates of a New Thiacarbocyanine Cy5 Dye for the Far-Red Spectral Region-a Spectroscopic and Cryo-Transmission Electron Microscopy Study. Phys. Chem. Chem. Phys. 2018, 20 (28), 18969-18977.

(36) Berlepsch, H. V; Böttcher, C. Cryo-Transmission Electron Microscopy Reveals Mesoscopic H-and J-Aggregates of near Infrared Cyanine Dyes. J. Photochem. Photobiol. A Chem. 2010, 214, 16-21. 
(37) Vitukhnovsky, A. G.; Lobanov, A. N.; Pimenov, A.; Yonezawa, Y.; Kometani, N.; Asami, K.; Yano, J. Absorption Spectra Simulation of Amalgamation-Type Cyanine Dye Aggregates in LB Films. J. Lumin. 2000, 87 (89), 260-262.

(38) Clark, K. A.; Krueger, E. L.; Vanden Bout, D. A. Direct Measurement of Energy Migration in Supramolecular Carbocyanine Dye Nanotubes. J. Phys. Chem. Lett. 2014, 5 (13), 2274-2282.

(39) Caram, J. R.; Doria, S.; Eisele, D. M.; Freyria, F. S.; Sinclair, T. S.; Rebentrost, P.; Lloyd, S.; Bawendi, M. G. Room-Temperature Micron-Scale Exciton Migration in a Stabilized Emissive Molecular Aggregate. Nano Lett. 2016, 16 (11), 6808-6815.

(40) Wang, C.; Weiss, E. A. Accelerating FRET between Near-Infrared Emitting Quantum Dots Using a Molecular J-Aggregate as an Exciton Bridge. Nano Lett. 2017, 17 (9), 5666-5671.

(41) Eisele, D. M.; Berlepsch, H. V.; Böttcher, C.; Stevenson, K. J.; Vanden Bout, D. A.; Kirstein, S.; Rabe, J. P. Photoinitiated Growth of Sub-7 Nm Silver Nanowires within a Chemically Active Organic Nanotubular Template. J. Am. Chem. Soc. 2010, 132 (7), 2104-2105.

(42) Freyria, F. S.; Cordero, J. M.; Caram, J. R.; Doria, S.; Dodin, A.; Chen, Y.; Willard, A. P.; Bawendi, M. G. Near-Infrared Quantum Dot Emission Enhanced by Stabilized Self-Assembled J-Aggregate Antennas. Nano Lett. 2017, 17 (12), 7665-7674.

(43) Scheblykin, I. G.; Sliusarenko, O. Y.; Lepnev, L. S.; Vitukhnovsky, A. G.; Auweraer, M. Van Der. Excitons in Molecular Aggregates of 3,3'-Bis-[3-Sulfopropyl]-5,5'-Dichloro-9Ethylthiacarbocyanine (THIATS): Temperature Dependent Properties. J. Phys. Chem. B 2001, 105, $4636-4646$.

(44) Scheblykin, I. G.; Bataiev, M. M.; Van der Auweraer, M.; Vitukhnovsky, A. G. Dimensionality and Temperature Dependence of the Radiative Lifetime of J-Aggregates with Davydov Splitting of the Exciton Band. Chem. Phys. Lett. 2000, 316 (1-2), 37-44. 
(45) Anantharaman, S. B.; Stöferle, T.; Nüesch, F. A.; Mahrt, R. F.; Heier, J. Exciton Dynamics and Effects of Structural Order in Morphology-Controlled J-Aggregate Assemblies. Advanced Functional Materials. John Wiley \& Sons, Ltd December 20, 2018, p 1806997.

(46) Misawa, K.; Ono, H.; Minoshima, K.; Kobayashi, T. New Fabrication Method for Highly Oriented J Aggregates Dispersed in Polymer Films. Appl. Phys. Lett. 1993, 63 (5), 577-579.

(47) Eisele, D. M.; Cone, C. W.; Bloemsma, E. A.; Vlaming, S. M.; van der Kwaak, C. G. F.; Silbey, R. J.; Bawendi, M. G.; Knoester, J.; Rabe, J. P.; Vanden Bout, D. A. Utilizing Redox-Chemistry to Elucidate the Nature of Exciton Transitions in Supramolecular Dye Nanotubes. Nat. Chem. 2012, 4 (8), 655-662.

(48) Takahashi, D.; Oda, H.; Izumi, T.; Hirohashi, R. Substituent Effects on Aggregation Phenomena in Aqueous Solution of Thiacarbocyanine Dyes. Dye. Pigment. 2005, 66 (1), 1-6.

(49) Didraga, C.; Pugžlys, A.; Hania, P. R.; Von Berlepsch, H.; Duppen, K.; Knoester, J. Structure, Spectroscopy, and Microscopic Model of Tubular Carbocyanine Dye Aggregates. J. Phys. Chem. B 2004, 108 (39), 14976-14985.

(50) Czikklely, V.; Forsterling, H. D.; Kuhn, H. Extended Dipole Model for Aggregates of Dye Molecules. Chem. Phys. Lett. 1970, 6 (3), 207-210.

(51) Czikkely, V.; Försterling, H. D.; Kuhn, H. Light Absorption and Structure of Aggregates of Dye Molecules. Chem. Phys. Lett. 1970, 6 (1), 11-14.

(52) Pandya, R.; Chen, R. Y. S.; Cheminal, A.; Thomas, T.; Thampi, A.; Tanoh, A.; Richter, J.; Shivanna, R.; Deschler, F.; Schnedermann, C.; Rao, A. Observation of Vibronic-Coupling-Mediated Energy Transfer in Light-Harvesting Nanotubes Stabilized in a Solid-State Matrix. J. Phys. Chem. Lett. 2018, 9 (18), 5604-5611. 
(53) Mukamel, S. Principles of Nonlinear Optical Spectroscopy; Oxford University Press: New York, 1995.

(54) Heijs, D. J.; Malyshev, V. A.; Knoester, J. Thermal Broadening of the J-Band in Disordered Linear Molecular Aggregates: A Theoretical Study. J. Chem. Phys. 2005, 123 (14), 144507.

(55) Ma, J.; Cao, J. Förster Resonance Energy Transfer, Absorption and Emission Spectra in Multichromophoric Systems. I. Full Cumulant Expansions and System-Bath Entanglement. J. Chem. Phys. 2015, 142 (9), 094106.

(56) Yamagata, H.; Spano, F. C. Interplay between Intrachain and Interchain Interactions in Semiconducting Polymer Assemblies: The HJ-Aggregate Model. J. Chem. Phys. 2012, 136 (18), 184901.

(57) Hestand, N. J.; Spano, F. C. Expanded Theory of H-and J-Molecular Aggregates: The Effects of Vibronic Coupling and Intermolecular Charge Transfer. 2018.

(58) Chuang, C.; Bennett, D. I. G.; Caram, J. R.; Aspuru-Guzik, A.; Bawendi, M. G.; Cao, J. Generalized Kasha's Scheme for Classifying Two-Dimensional Excitonic Molecular Aggregates: Temperature Dependent Absorption Peak Frequency Shift. Arxiv 2019, arXiv:1901.01318v1.

(59) Kaiser, T. E.; Scheblykin, I. G.; Thomsson, D.; Wu, F. Temperature-Dependent Exciton Dynamics in J-AggregatessWhen Disorder Plays a Role. J. Phys. Chem. B 2009, 15836-15842.

(60) Scholes, G. D.; Fleming, G. R.; Olaya-Castro, A.; van Grondelle, R. Lessons from Nature about Solar Light Harvesting. Nat. Chem. 2011, 3 (10), 763-774.

(61) Nugraha, A. R. T.; Saito, R.; Sato, K.; Araujo, P. T.; Jorio, A.; Dresselhaus, M. S. Dielectric Constant Model for Environmental Effects on the Exciton Energies of Single Wall Carbon Nanotubes. Appl. Phys. Lett. 2010, 97 (9), 091905. 
1 (62) Ferretti, M.; Hendrikx, R.; Romero, E.; Southall, J.; Cogdell, R. J.; Novoderezhkin, V. I.; Scholes, G. D.; Van Grondelle, R. Dark States in the Light-Harvesting Complex 2 Revealed by TwoDimensional Electronic Spectroscopy. Sci. Rep. 2016, 6 (1), 20834.

(63) Gao, X.; Eisfeld, A. Near-Field Spectroscopy of Nanoscale Molecular Aggregates. J. Phys. Chem. Lett. 2018, 9 (20), 6003-6010.

(66) Lambert, C.; Koch, F.; Völker, S. F.; Schmiedel, A.; Holzapfel, M.; Humeniuk, A.; Röhr, M. I. S.;

(67) Saikin, S. K.; Eisfeld, A.; Valleau, S.; Aspuru-Guzik, A. Photonics Meets Excitonics: Natural and Artificial Molecular Aggregates. Nanophotonics 2013, 2 (1), 21-38. 


\title{
Supporting Information (SI) for
}

\section{Design Principles for 2-Dimensional Molecular Aggregates using Kasha's Model: Tunable Photophysics in Near and Shortwave Infrared}

\author{
Arundhati P. Deshmukh ${ }^{1}$, Danielle Koppel ${ }^{1}$, Chern Chuang ${ }^{2}$, Danielle M. Cadena, ${ }^{1}$ Jianshu Cao ${ }^{3}$ \\ and Justin R. Caram ${ }^{1 *}$ \\ ${ }^{1}$ Department of Chemistry and Biochemistry, University of California, Los Angeles, 607 Charles E. Young \\ Dr. East, Los Angeles, California-90095, United States. ${ }^{2}$ Department of Chemistry, University of Toronto, \\ 80 St. George Street, Toronto, Ontario M5S 3H6, Canada. ${ }^{3}$ Department of Chemistry, Massachusetts \\ Institute of Technology, Cambridge, Massachusetts 02139, USA
}

Email: jcaram@chem.ucla.edu 


\section{Table of Contents}

\section{Section}

Page no.

1. Aggregation of Cy7-DPA and Cy7-Ph in methanol-water mixtures

2. Structural characterization of the aggregates

2.1 Dynamic light scattering $\quad 4$

2.2 Concentration dependence of H-aggregate 5

3. Modeling aggregate photophysics

3.1 Extended dipole model $\quad 6$

3.2 Frenkel Exciton Hamiltonian and Density of States (DOS) 7

$\begin{array}{ll}3.3 \text { Bright state energy and DOS } & 8\end{array}$

3.4 Approximating extended dipole model parameters for Cy7-DPA and Cy7-Ph 9

$\begin{array}{ll}\text { 4. Sugar matrix stabilization and temperature dependence } & 10\end{array}$

5. Effect of dimensionality on band structure 11

6. Estimating the extent of exciton delocalization 12 


\section{Aggregation of Cy7-DPA and Cy7-Ph in methanol-water mixtures}

We form a monomer stock solution by dissolving the dyes in methanol. We then mix the monomer solution with deionized water in varying volume/volume fractions. We keep the final dye concentration constant modifying the concentration of stock solution accordingly. Fig. S1a-b show the variation of the aggregate and monomer peaks (from main text Fig. 2a-b) for the whole range of \%MeOH.

We obtain an average transition dipole moment (TDM, $\mu$ ) from the absorption spectra for both the dyes using equations $1 \mathrm{a}$ and $1 \mathrm{~b}$ :

$$
f=4.3 \times 10^{-9} \int \varepsilon d \bar{v} \quad(1 \mathrm{a}), \quad\langle\mu\rangle^{2}=\frac{3 h e^{2}}{8 \pi^{2} m \bar{v}} f
$$

where $\mathrm{f}$ is the oscillator strength, $\varepsilon$ is the molar absorptivity, $\bar{v}$ is the peak wavenumber, $m$ and $e$ are the mass and charge of an electron and $h$ is Planck's constant. ${ }^{1,2}$ The average TDMs were 4.2 +/- $0.9 \mathrm{D}$ and 4.0 +/- 0.5 D for Cy7-DPA and Cy7-Ph respectively.

a

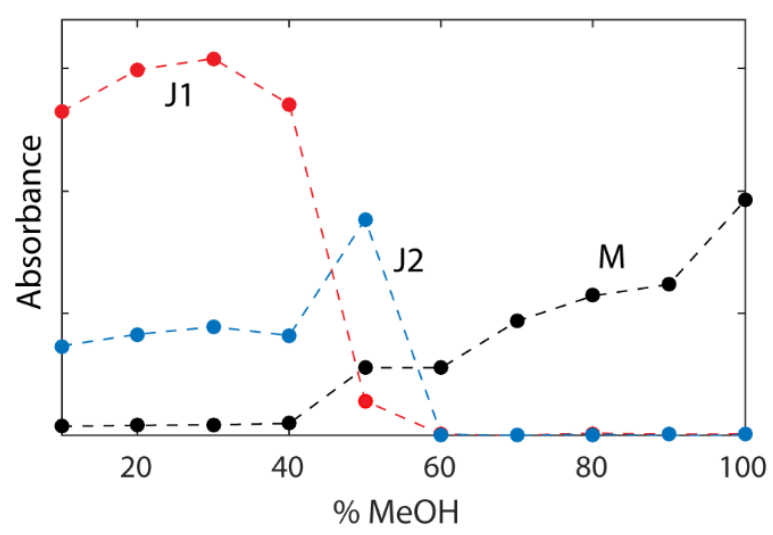

b

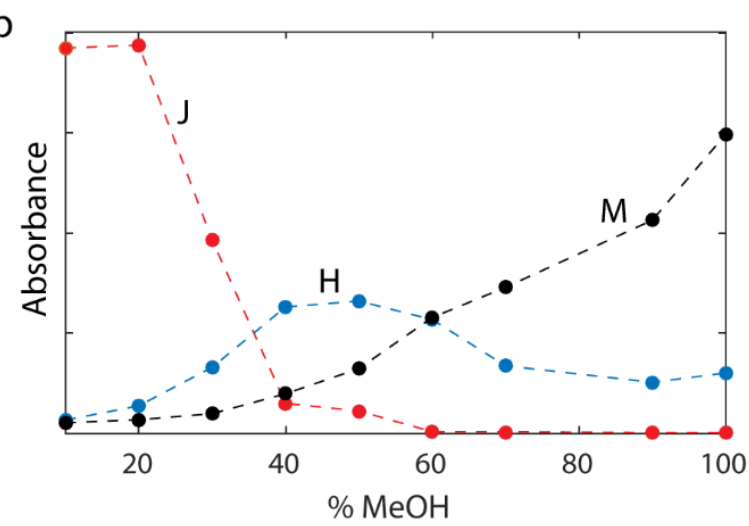

Figure S1: Peak values for each aggregate and monomer peak (from main text Fig. 2a-b) as a function of \%MeOH (0-100\%) for (a) Cy7-DPA and (b)Cy7-Ph. 


\section{Structural Characterization of the aggregates}

\subsection{Dynamic Light Scattering (DLS)}

Cryo-electron microscopy reveals sheet-like morphologies for Cy7-DPA and Cy7-Ph aggregates with widths varying from $\sim 100-300 \mathrm{~nm}$ and lengths up to micron scale. Sheets of Cy7-Ph appear to be longer than C7-Ph. For a bulk estimate of aggregate sizes, we performed dynamic light scattering (DLS) on the Jaggregates of $\mathrm{Cy} 7-\mathrm{DPA}$ and $\mathrm{Cy} 7-\mathrm{Ph}$. We approximate particle sizes from the intensity distribution following the treatment done by Lotya et. al. ${ }^{3}$ The particle size estimates are shown in Fig. S2 and agree well with the aggregate extent shown in cryoEM images.
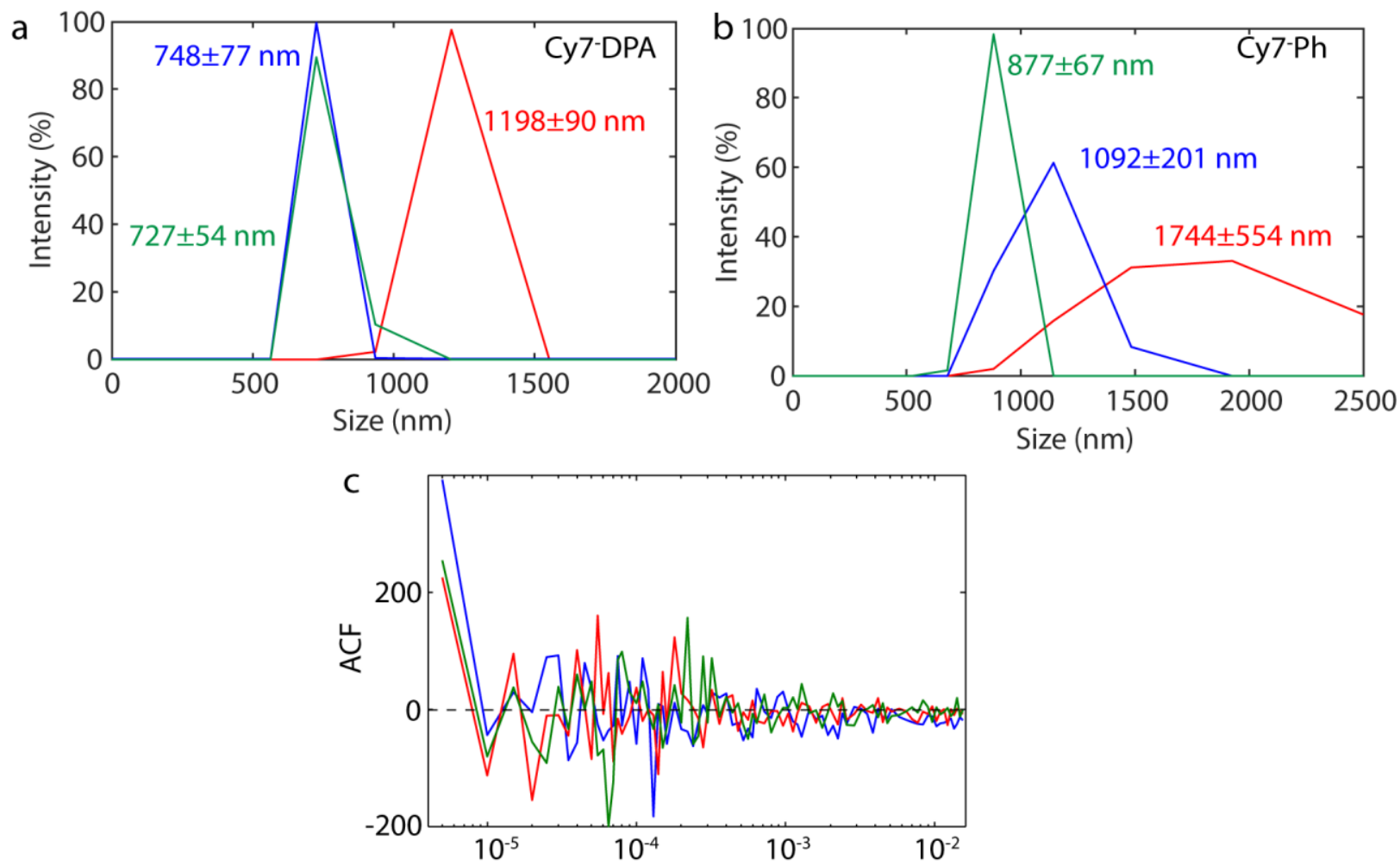

Figure S2: DLS intensity distributions for (a) Cy7-DPA and (b) Cy7-Ph, 20\% MeOH aggregate samples. (c) Auto-correlation function (ACF) of the solvent mixture $(20 \% \mathrm{MeOH})$ showing no contribution coming from the mixture. Red, green and blue curves represent different trials. 


\subsection{Concentration dependence of $\mathrm{H}$-aggregate}

To assign the $\mathrm{H}$-aggregate peak in Cy7-Ph (Fig. 2a from main text), we observed concentration dependence of the $70 \% \mathrm{MeOH}$ sample. ${ }^{4}$ In this case, the $\% \mathrm{MeOH}$ was kept constant while the total concentration of the dye was changed from $0.05-0.5 \mathrm{mM}$. The relative changes in the peak absorbances for monomer and $\mathrm{H}$-aggregate peaks were used to obtain the number of monomers in the $\mathrm{H}$-aggregate (dimer $\mathrm{v} / \mathrm{s}$ extended aggregate) using the following set of equations:

$$
\begin{gathered}
n M \rightleftharpoons H \\
K=\frac{C_{H}}{\left(C_{M}\right)^{2}}
\end{gathered}
$$

Taking log and rearranging eqn. (2b),

$$
\log \left(C_{H}\right)=\log (K)+\mathrm{n} * \log \left(C_{M}\right)
$$

where $M$ and $H$ denote the monomer and $\mathrm{H}$-aggregate respectively. $K$ is the equilibrium constant and $C$ denotes the concentration of the species. $C_{M}$ can be obtained from the absorption co-efficient of the monomer, $C_{M}=A b s_{M} /\left(\varepsilon_{M} \cdot l\right)$. The absorption co-efficient for the monomer was found to be $3.82 \times 10^{5}$ $\mathrm{M}^{-1} \mathrm{~cm}^{-1}$. Although the absorption co-efficient of the $\mathrm{H}$-aggregate cannot be accurately determined, we can use the absorbance of the $\mathrm{H}$-aggregate $\left(A b s_{H}\right)$ as a proxy. Since $A b s_{H} \propto C_{H}$, the proportionality constant would simply change the intercept of eqn. (2c). Using this approximation, we obtain eqn. (2d):

$$
\begin{gathered}
\log \left(A b s_{H}\right)-\log \left(\left(\varepsilon_{H} \cdot l\right)\right)=\log (K)+\mathrm{n} * \log \left(C_{M}\right) \\
\log \left(A b s_{H}\right)=\log \left(K^{\prime}\right)+\mathrm{n} * \log \left(C_{M}\right)
\end{gathered}
$$

where $K^{\prime}=K /\left(\varepsilon_{H} \cdot l\right)$ is a constant. This equation is only valid for the slope. To obtain the equilibrium constant accurately, we will need to determine the concentration of the $\mathrm{H}$-aggregated species. ${ }^{4}$ Fig. S3 shows a plot of $\log \left(A b s_{H}\right) \mathrm{v} / \mathrm{s} \log \left(C_{M}\right)$, the linear fit gives $n=1.5(0.7,2.3)$. This means that the $\mathrm{H}$ aggregate peak at $698 \mathrm{~nm}\left(14326 \mathrm{~cm}^{-1}\right)$ can be assigned to H-dimer.

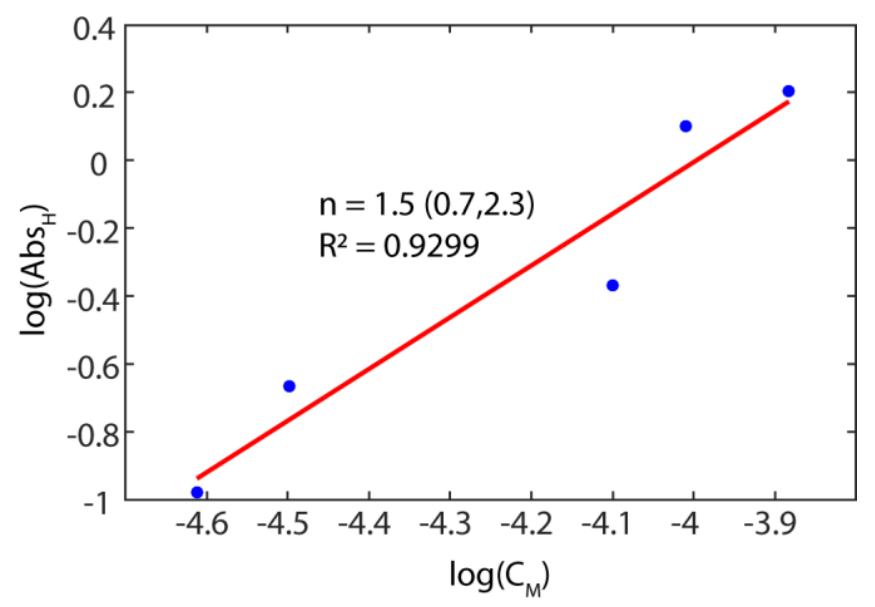

Figure S3: Plot of $\log \left(\mathrm{Abs}_{\mathrm{H}}\right)$ against $\log \left(\mathrm{C}_{\mathrm{M}}\right)$ for $\mathrm{H}$-aggregate samples $(70 \% \mathrm{MeOH})$ with varying final concentration $(0.05,0.1$, $0.2,0.5$ and $0.5 \mathrm{mM})$. Straight line denotes a linear fit to equation (2e). 


\section{Modeling aggregate photophysics}

\subsection{Extended dipole model}

We adapt the Frenkel exciton theory for modeling the aggregates. ${ }^{5,6}$ We start with an extended dipole model where we treat a molecule as rectangular brick with side lengths represented by $a$ (short side) and $b$ (long side) as shown in Fig. S4. Along the long axis, we place partial charges of equal and opposite charge, equidistant from the center. From this charge separation, we calculate the magnitude of the transition dipole moment (TDM) on a single brick generated with one excitation. We build a sheet of similar bricks separated by the parameters $a$ and $b$, where the nearest bricks are 'slipped' with respect to each other by a slip parameter $s$. Each brick is callable with a specific label or index. We use monolayer sheet in our model as the it's unlikely to affect the overall photophysics as suggested by previous literature. ${ }^{7}$

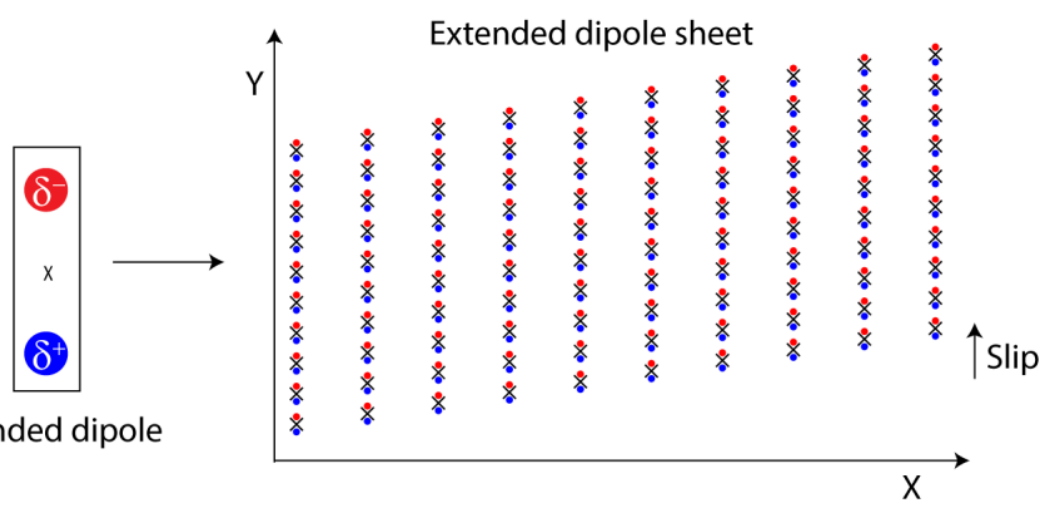

Figure S4: Schematic depiction (not to scale) of a magnified extended dipole brick (left) and a 2-D aggregate sheet constructed from slipped arrangement of the bricks (right). 


\subsection{Frenkel Exciton Hamiltonian and Density of States (DOS)}

Next, we construct the Frenkel exciton Hamiltonian from the electrostatic interaction between the TDMs. Represented in the site basis, the Hamiltonian is a matrix of dimensions $n \times n$, where $n$ is the total number of bricks. Diagonal elements represent individual site energies and off-diagonal elements represent coupling energies obtained from equation 3:

$$
J_{\text {ext }, n, m} \sim \frac{\mu^{2}}{d}\left(\frac{1}{r_{m+n+}}-\frac{1}{r_{m+n-}}-\frac{1}{r_{m-n+}}+\frac{1}{r_{m-n-}}\right)
$$

where $\mu$ is the TDM per site, $d$ is the charge distance per site and $r_{m \pm n \pm}$ is the distance between $m^{\text {th }}$ and $n^{\text {th }}$ charge.

As an example, we represent a coupling matrix for a $6 \times 6$ brick lattice (Fig. S5). As expected, nearestneighbor coupling is the largest contribution. We go on to add inhomogeneous site disorder. With an open variance parameter, we draw from a normal distribution centered around the monomer energy. A variance of zero results in all sites with identical energy (same chemical environments). A nonzero variance shifts each monomer by an individually chosen amount as shown by varying diagonal elements. To eliminate edge effects, we add periodic boundary conditions (PBC) that couple the initial aggregate to repeated images of the aggregate along both major axes. In the limit of large site numbers, edge effects become less significant. In our modeling (shown later in section 6) we find the delocalization length is limited to 8 sites. Introducing the PBC and, therefore, eliminating the edge effects can be justified.

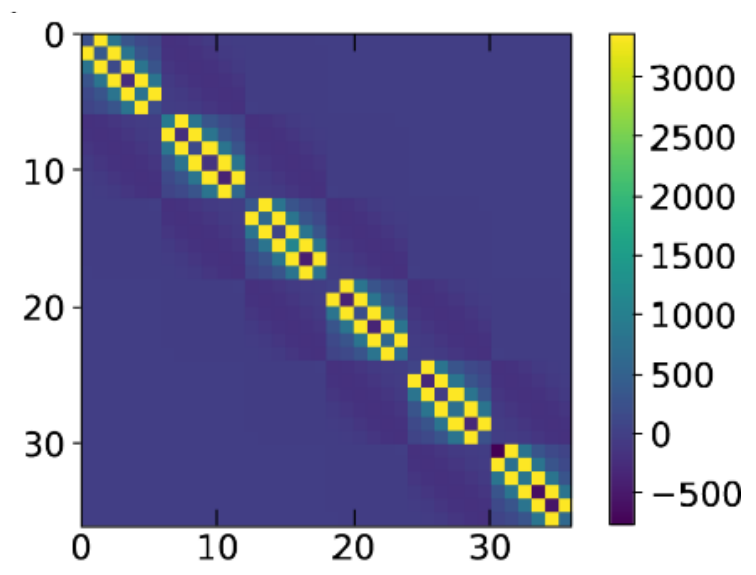

Figure S5: Example coupling matrix for a 6x6 aggregate. 


\subsection{Bright state energy and DOS}

Diagonalizing the $n \times n$ coupling Hamiltonian produces $n$ exciton energies corresponding to $n$ exciton states (Fig. S6). These eigenvectors are stationary exciton states, which can be written as a linear superposition of the states in the site basis. We then go on to obtain the density of states (DOS) by histogramming all the eigenvalues (energies) obtained from the Hamiltonian (main text Fig. 3b). The optically active state (bright state) requires a net non-zero TDM as per Fermi's Golden Rule. ${ }^{2}$ The net TDM per exciton was calculated as the sum of delocalization coefficients $c_{l}$ (from eigenvectors) squared,

$$
\mu_{k}=\left|\sum_{l=1}^{n} c_{k, l}\right|^{2}
$$

Summing before squaring accounts for the phase relationship between TDMs of each site. We obtain the absorption spectra by histogramming eigenvalues weighed by with their respective net TDM.

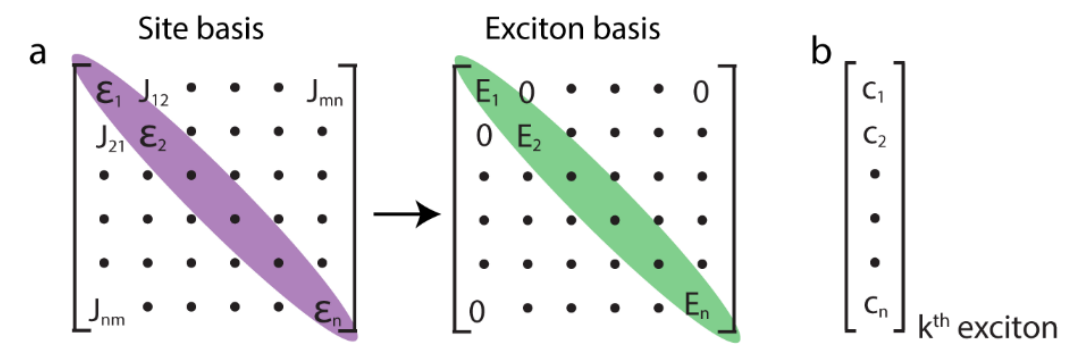

Figure S6: (a) Coupling matrix in site basis and its diagonalization to the exciton basis, (b) Example eigenvector for $\mathrm{k}^{\text {th }}$ exciton where the coefficients correspond to the extent of delocalization over each site. 


\subsection{Approximating extended dipole model parameters for Cy7-DPA and Cy7-Ph}

To model Cy7-DPA and Cy7-Ph, we obtain the transition charge densities of both dyes using a semiempirical method in the software ZINDO/S. ${ }^{8}$ The dyes were considered without the sulfonyl chains and with halogen groups replaced with methyl groups for simplicity. For an isolated monomer in vacuum, we optimize a ground state configuration using molecular modeling on Spartan and use it as an initial condition for ZINDO. We obtain many excited state transitions along with their net TDM, atomic coordinates of the excited state and the transition charges per atom. We select the transition with highest TDM as it represents the highest transition probability. Since the coupling calculation for many-dye systems is too computationally expensive with all charges considered, we fit extended dipole parameters for a dimer system to the dimer coupling calculated from transition charges. To start, we construct a dimer using the calculated transition charges obtained from ZINDO and compute coupling as a function of slip using the following eqn:

$$
J_{\text {dimer }} \sim \sum_{i=1}^{n} \sum_{j=1}^{n} \frac{q_{i} q_{j}}{r_{i j}}=\sum_{i=1}^{n} \sum_{j=1}^{n} \frac{q_{i} q_{j}}{\sqrt{\left(x_{i}-x_{i}\right)^{2}+\left(y_{i}-y_{i}+s\right)^{2}}}
$$

where the numerator is the product of charges (one from each dye) and the denominator is the distance between these charges, $n$ is the total number of transition charges per dye, and $s$ is the slip. The total TDM per dye was calculated using the transition charge densities and distances relative to an arbitrary origin atom following eqn:

$$
\mu=\sum_{i=1}^{n} q_{i} r_{i, 0}
$$

We scale this TDM to match experimental values obtained from the absorption spectra shown in Fig. S1. We construct an extended dipole dimer with the scaled TDM and brick dimensions extracted from the atomic coordinates and an open charge distance parameter. We calculate the coupling for this dimer as a function of slip using eqn. (3). We vary the parameter $d$ (charge distance) until the two curves match to obtain a reasonable charge distance (Fig. S7). The agreement is not the best for the entire range of slip values, but it fits well for smaller slip values which is where we expect our systems to be.

We average the parameters of both dyes and use them to calculate the coupling matrices for $20 \times 20$ sheets at various slips. The complex dielectric environment in the aggregates gives rise to large long range coupling among monomer units. We approximate the dielectric environment by the scaling the coupling matrix by a factor of 70.54. This scaling factor is an approximation of the dielectric constant (weighted sum of dielectric constants of methanol and water). A more accurate description of the dielectric environment is outside the scope of this paper."
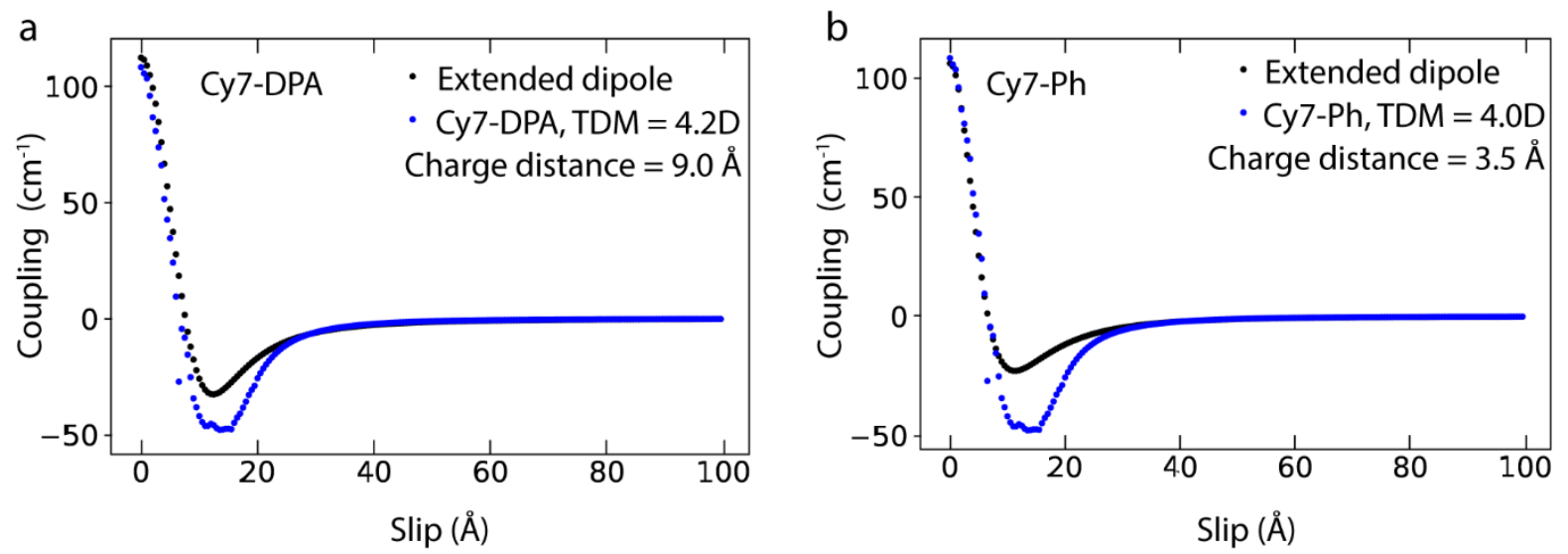

Figure S7: Fitting out the extended dipole parameters from transition charge density coupling calculations of dimers for (a) Cy7DPA and (b) Cy7-Ph 


\section{Sugar matrix stabilization and temperature dependence}

We prepare the stabilized aggregates using a previously reported sugar matrix stabilization procedure. ${ }^{9}$ Matrix stabilization allows the aggregates to maintain their morphology while keeping the samples optically transparent at low temperatures. Fig. S8a shows the absorption spectra of solution and sugar matrix stabilized aggregates. Sugar matrix stabilization does not affect the absorption of Cy7-DPA at all. It does, however introduce some disorder in Cy7-Ph as seen from the broadened absorption. Nevertheless, the aggregates morphology is still unaffected as the peak doesn't shift. Fig. S8b shows the peak position as a function of temperature. We observe a large red-shift for Cy7-DPA upon cooling, Cy7Ph shows a slight blue-shift.

a

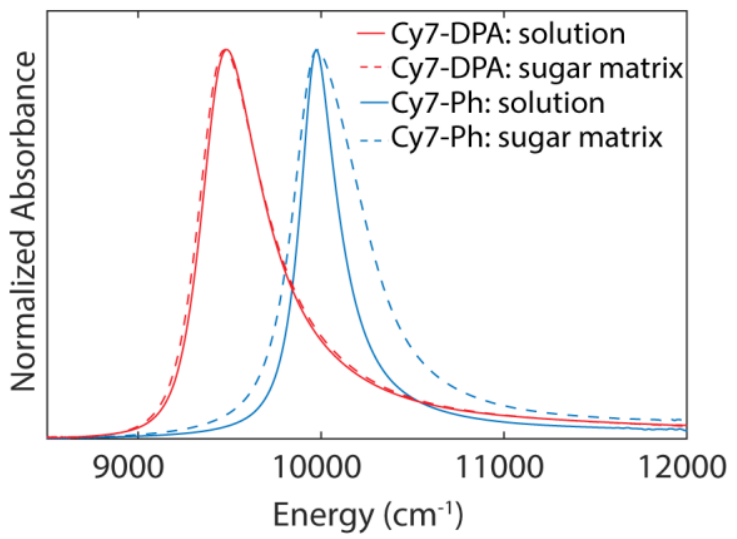

b

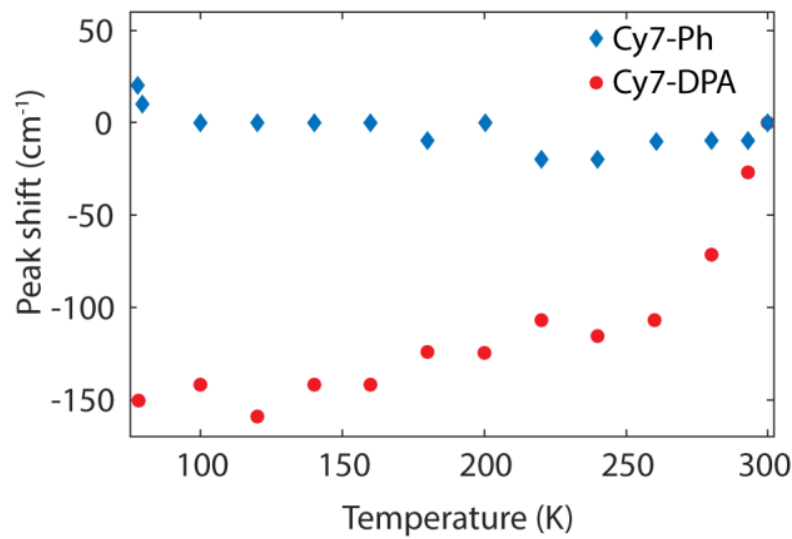

Figure S8: (a) Absorption spectra of Cy7-DPA (red) and Cy7-Ph (blue) aggregates in solution (solid line) and sugar matrix (dashed lines); (b) Peak shift as a function of temperature for Cy7-DPA (red circles) and Cy7-Ph (blue diamonds) with respect to the peak position at $300 \mathrm{~K}$. Negative (positive) values indicate red (blue) shift. 


\section{Effect of dimensionality on band structure}

In Fig. S9, we plot the eigenvalues of the Hamiltonian against the number of nodes in that eigenstate as a function of slip (each pair of sequential out-of-phase TDMs was considered a node). Disorder was not included in these calculations, nor were non-dipole-dipole forms of coupling (e.g. quadrupole or chargetransfer) and which can change the band-structure. The bright state always has zero nodes $\left(\mathrm{k}_{\mathrm{x}}, \mathrm{k}_{\mathrm{y}}=0\right)$. Here, we have assumed a finite size of the aggregate and the dielectric scaling factor (described in section 3.4). While the finite size of the aggregates will cause some redistribution of the oscillator strength around the bright state, ${ }^{10,11}$ we observe the phenomenon of I-aggregation in the $2 \mathrm{D}$ aggregate. In the $1 \mathrm{D}$ case shown in Fig. S9a, the bright state is always on either side of the band-edge (highest or lowest eigenvalue) for slips. Contrastingly, in the 2D cases shown in Fig. S9c-d, the bright state still maintains its zero node identity but it is not restricted to the band-edge depending on the slip value. This means that the dark states in I-aggregates may potentially couple to other transitions via near-field coupling.

a

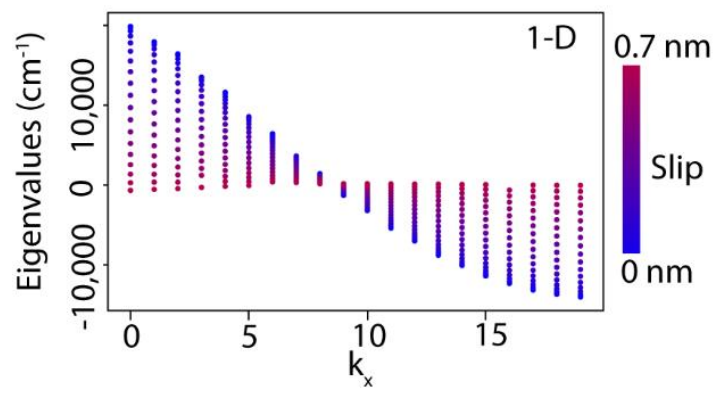

C

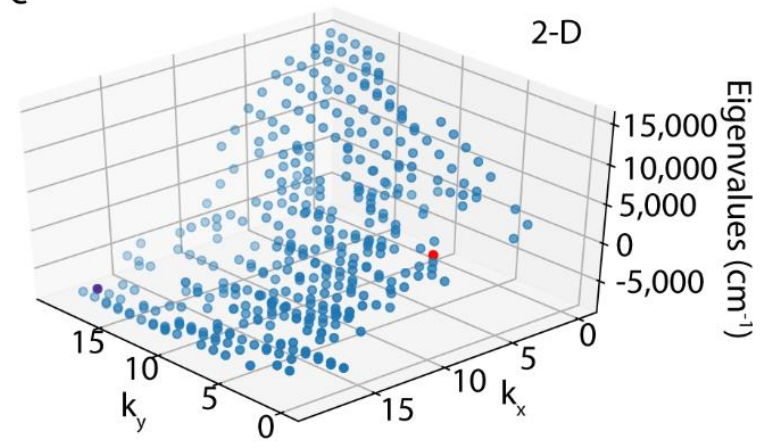

b

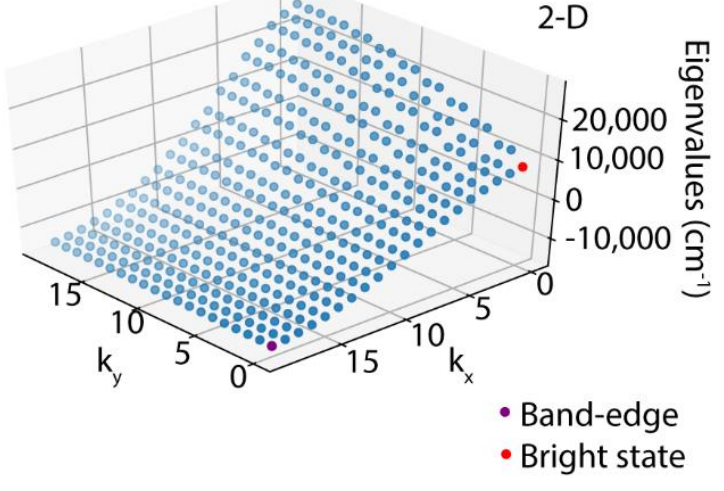

$\mathrm{d}$

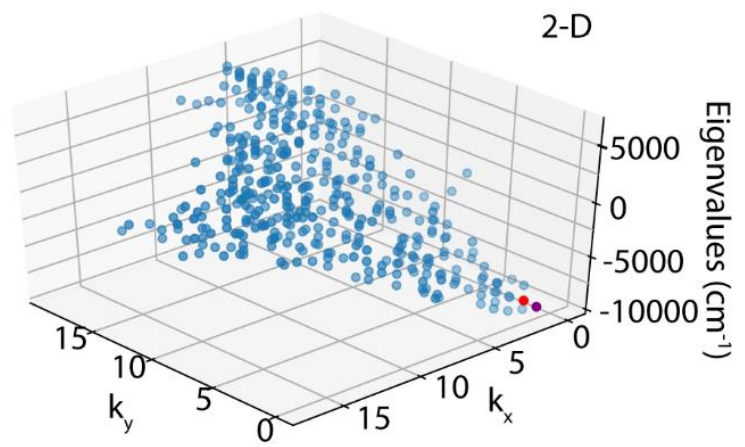

Figure S9: Eigenvalues of the aggregate Hamiltonian (with no disorder) as a function of number of nodes ( $\left.k_{x}, k_{y}\right)$ for (a) 1-D aggregate with increasing slip (blue $=0 \mathrm{~nm}$, red=0.7 nm); (b), (c) and (d) 2-D aggregate with $0 \mathrm{~nm}, 0.4 \mathrm{~nm}$ and $0.7 \mathrm{~nm}$ slips respectively. 


\section{Estimating the extent of exciton delocalization}

The number of sites an exciton is delocalized over can be calculated as the inverse participation ratio $(I)$, which is the inverse sum of the scaling coefficients raised to the power of four (eqn 7a). We average $I$ over many realizations of the Hamiltonian with varying disorder to estimate the extent of exciton delocalization. In Fig. S10, we show the inverse participation ratio $I$ and linewidth $W$ of the absorption spectra for a 20x28 aggregate with increasing site disorder. $I$ is calculated and averaged over disorder realizations via

$$
\begin{aligned}
& I_{k}=\sum_{l=1}^{n} \frac{1}{\left|c_{k, l}\right|^{4}} \\
& \mu_{k}=\left|\sum_{l=1}^{n} c_{k, l}\right|^{2} \\
& I_{a g g}=\frac{1}{n} \sum_{k=1}^{n} \mu_{k} * I_{k} \\
& \left\langle I_{\text {agg }}\right\rangle=\frac{1}{N} \sum_{l=1}^{N} I_{\text {agg }}
\end{aligned}
$$

The linewidth is calculated by assuming that the absorption spectra can be decomposed into individual eigenstates, each with a Lorentzian profile with width determined by the dephasing rate $W_{k}$ as in Eq.(3) of the main text:

$$
A(\omega)=\sum_{k=1}^{n} \mu_{k}^{2} \cdot \frac{W_{k} / 2}{\left(\omega-\epsilon_{k}\right)^{2}+\left(W_{k} / 2\right)^{2}}
$$

We determine slip $=4.0 \AA$ to be the best fit for the Cy7-Ph I-aggregate basing on the temperaturedependent absorption spectra. And the bath spectral density is fitted from the monomer absorption lineshape to an Ohmic bath with exponential cut-off.

$D_{b}(E)=\pi \lambda \cdot \frac{\omega}{\omega_{c}} e^{-\omega / \omega_{c}}$

where $\lambda=600 \mathrm{~cm}^{-1}$ and $\omega_{\mathrm{c}}=1000 \mathrm{~cm}^{-1}$.
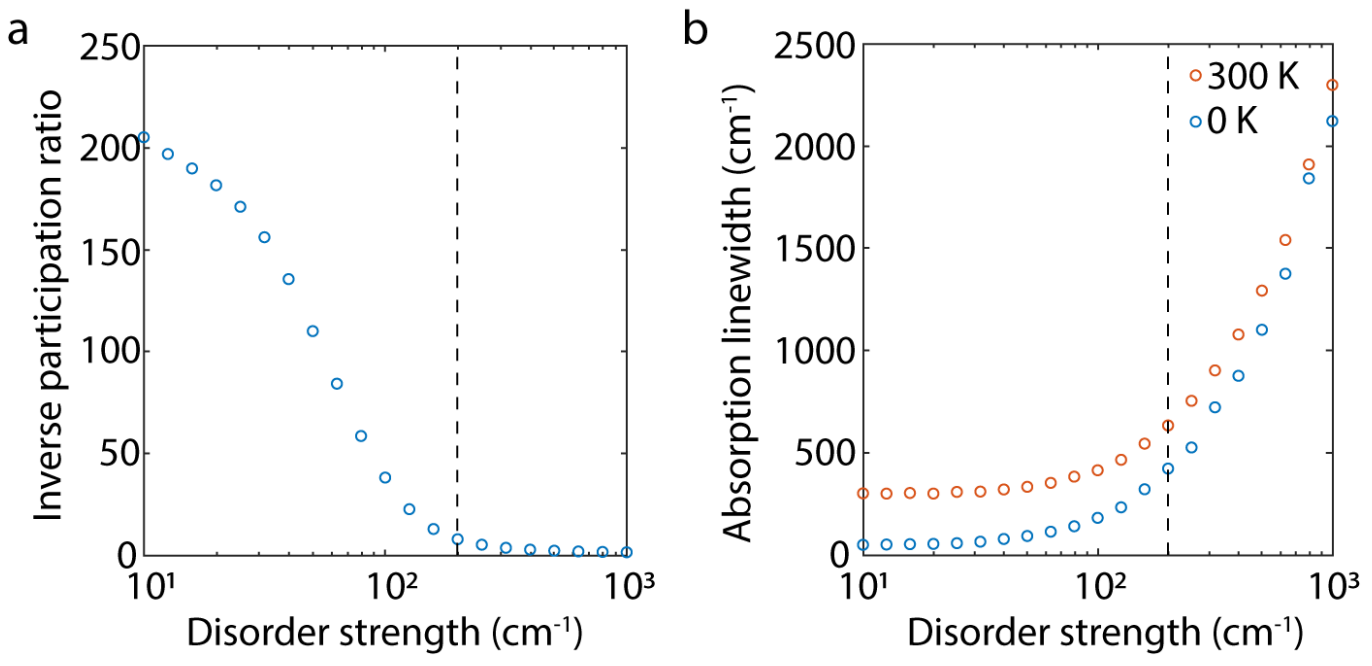

Figure S10: (a) Inverse participation ratio (number of sites the exciton is delocalized over) and (b) Linewidths for a $20 \times 28$ aggregate as a function of site disorder. Dotted lines indicate experimental linewidth. 
As expected, I decreases and linewidth increases with increasing disorder. This set of parameters slightly underestimates the contribution from the temperature-independent homogeneous linewidth $\left(W_{k}^{(0)}\right)$. We attribute the deviation to the inaccuracy of the extended dipole model for the short-range interactions among the dyes. We estimate that the excitons are delocalized over $\sim 8$ sites at room temperature.

\section{References}

(1) Hilborn, R. C. Einstein Coefficients, Cross Sections, f Values, Dipole Moments, and All That. Am. J. Phys. 1982, 50, 982.

(2) Turro, N. J.; Ramamurthy, V.; Scaiano, J. C. Principles of Molecular Photochemistry : An Introduction; University Science Books: Sausalito, 2009.

(3) Lotya, M.; Rakovich, A.; Donegan, J. F.; Coleman, J. N. Measuring the Lateral Size of Liquid-Exfoliated Nanosheets with Dynamic Light Scattering. Nanotechnology 2013, 24 (26), 265703.

(4) Takahashi, D.; Oda, H.; Izumi, T.; Hirohashi, R. Substituent Effects on Aggregation Phenomena in Aqueous Solution of Thiacarbocyanine Dyes. Dye. Pigment. 2005, 66 (1), $1-6$.

(5) Didraga, C.; Pugžlys, A.; Hania, P. R.; Von Berlepsch, H.; Duppen, K.; Knoester, J. Structure, Spectroscopy, and Microscopic Model of Tubular Carbocyanine Dye Aggregates. J. Phys. Chem. B 2004, 108 (39), 14976-14985.

(6) Czikklely, V.; Forsterling, H. D.; Kuhn, H. Extended Dipole Model for Aggregates of Dye Molecules. Chem. Phys. Lett. 1970, 6 (3), 207-210.

(7) Eisele, D. M.; Cone, C. W.; Bloemsma, E. A.; Vlaming, S. M.; van der Kwaak, C. G. F.; Silbey, R. J.; Bawendi, M. G.; Knoester, J.; Rabe, J. P.; Vanden Bout, D. A. Utilizing RedoxChemistry to Elucidate the Nature of Exciton Transitions in Supramolecular Dye Nanotubes. Nat. Chem. 2012, 4 (8), 655-662.

(8) Frisch, M. J.; Trucks, G. W.; Schlegel, H. B.; Scuseria, G. E.; Robb, M. A.; Cheeseman, J. R.; Scalmani, G.; Barone, V.; Petersson, G. A.; Nakatsuji, H.; Li, X.; Caricato, M.; Marenich, A. V; Bloino, J.; Janesko, B. G.; Gomperts, R.; Mennucci, B.; Hratchian, H. P.; Ortiz, J. V; Izmaylov, A. F.; Sonnenberg, J. L.; Williams-Young, D.; Ding, F.; Lipparini, F.; Egidi, F.; Goings, J.; Peng, B.; Petrone, A.; Henderson, T.; Ranasinghe, D.; Zakrzewski, V. G.; Gao, J.; Rega, N.; Zheng, G.; Liang, W.; Hada, M.; Ehara, M.; Toyota, K.; Fukuda, R.; Hasegawa, J.; Ishida, M.; Nakajima, T.; Honda, Y.; Kitao, O.; Nakai, H.; Vreven, T.; Throssell, K.; Montgomery Jr., J. A.; Peralta, J. E.; Ogliaro, F.; Bearpark, M. J.; Heyd, J. J.; Brothers, E. N.; Kudin, K. N.; Staroverov, V. N.; Keith, T. A.; Kobayashi, R.; Normand, J.; Raghavachari, K.; Rendell, A. P.; Burant, J. C.; Iyengar, S. S.; Tomasi, J.; Cossi, M.; Millam, J. M.; Klene, M.; Adamo, C.; Cammi, R.; Ochterski, J. W.; Martin, R. L.; Morokuma, K.; Farkas, O.; Foresman, J. B.; Fox, D. J. Gaussian 16 RR\}evision $\{B\} .01 .2016$.

(9) Caram, J. R.; Doria, S.; Eisele, D. M.; Freyria, F. S.; Sinclair, T. S.; Rebentrost, P.; Lloyd, S.; Bawendi, M. G. Room-Temperature Micron-Scale Exciton Migration in a Stabilized 
Emissive Molecular Aggregate. Nano Lett. 2016, 16 (11), 6808-6815.

(10) Müller, M.; Paulheim, A.; Eisfeld, A.; Sokolowski, M. Finite Size Line Broadening and Superradiance of Optical Transitions in Two Dimensional Long-Range Ordered Molecular Aggregates. J. Chem. Phys. 2013, 139 (4), 044302.

(11) Allec, S. I.; Ilawe, N. V; Wong, B. M. Unusual Bandgap Oscillations in Template-Directed ф-Conjugated Porphyrin Nanotubes. J. Phys. Chem. Lett. 2016, 7 (13), 2362-2367. 
